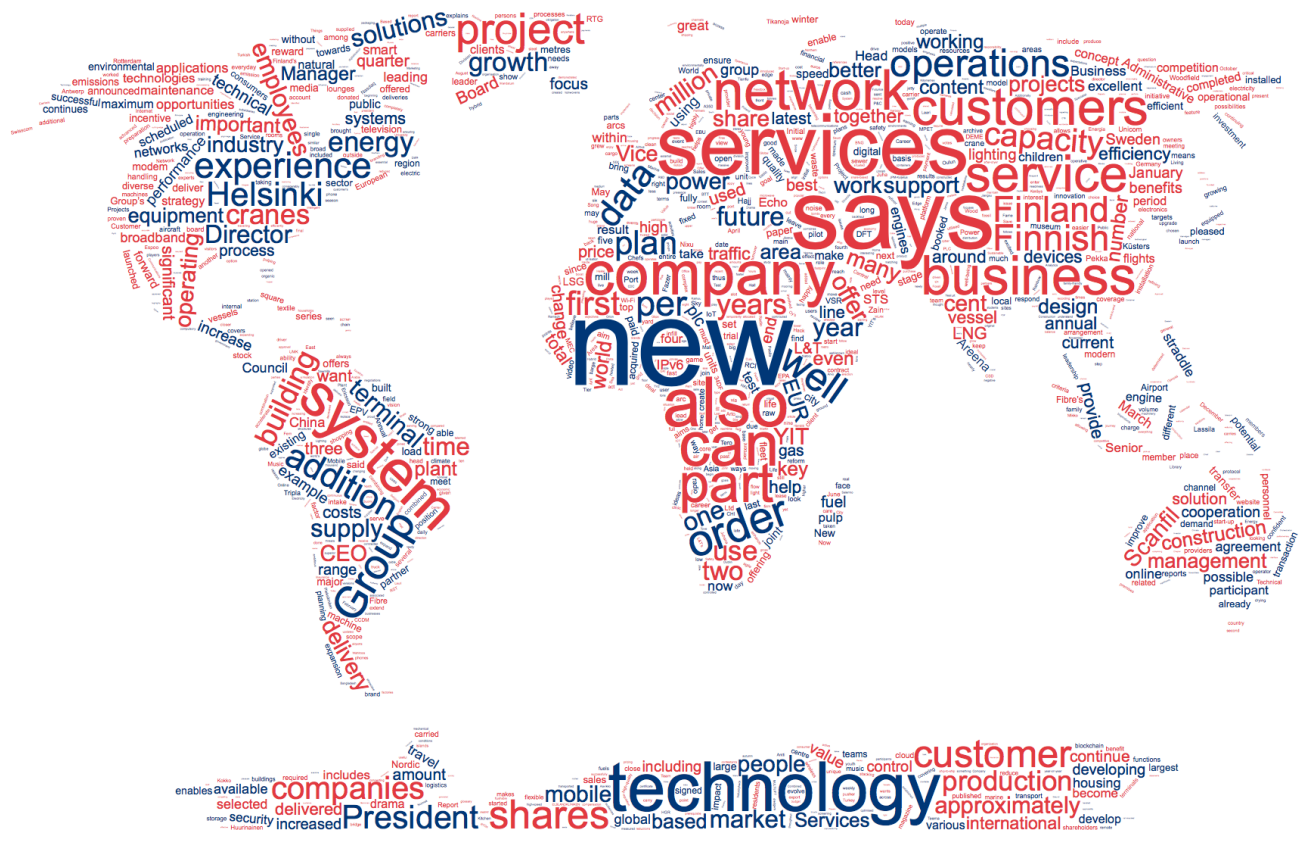

\title{
EXPLORATORY INVESTIGATION INTO THE PRACTICE OF COMMUNICATING TO PUBLICS USING ENGLISH AS A LINGUA FRANCA (ELF) BY FINNISH COMPANIES
}

Darren P. Ingram

Media and Communications Studies One-year Master's degree (15 credit thesis) Spring 2017

Supervisor: Prof. Dr. Bo Reimer

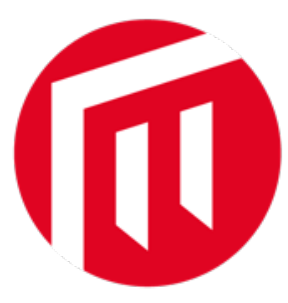




\begin{abstract}
This thesis examines public relations (PR) communications that use English as a Lingua Franca (ELF) from leading Finnish companies. It analyses a corpus of 90 press releases from 15 export-active companies for linguistic usage, drawing on elements of linguistic theory. In addition, a limited narrative is based on personal interviews to determine typical procedures that are involved in PR content creation. It should have relevance to all who use ELF in a business context, but be of special interest to those involved with PR and marketing. It may also have some relevance to internal international business communications and linguistics.
\end{abstract}

The study was motivated by three factors: how English is a dominant global language that is being used by companies in other countries as an intermediary language, prior research of how Finnish companies use PR and communications within their export activities, and extensive observation obtained whilst working as a journalist, dealing with companies from all around the world.

It is believed that many companies may not be communicating efficiently and effectively when using ELF. Even when they do communicate and content may appear to be grammatically correct, its efficacy may be muted, inhibiting audience comprehension and other consequential actions. The research noted that certain linguistic elements were over-represented, which could potentially inhibit communication and comprehension. The resolution is not necessarily drastic and could only deliver wider benefits where implemented.

Recommendations include closer attention is made concerning linguistic construction, broader additional research is conducted into the global phenomena and the possible creation of an operational framework to assist deployment of ELF-friendly textual communications, especially within the PR/marketing field.

KEYWORDS: business communication, globalization, English as a Lingua Franca (ELF), international business, linguistics, marketing, public relations (PR) 


\section{FOREWORD}

I continue to ride my hobby-horse about how too-many companies seem to be terrible at communicating. It seems that actions speak louder than words. If they communicate, you may struggle to understand what they want to say, particularly if they beat around the bush. This can be irrespective of whether a common language or a foreign language is used. Think about those who use a foreign language as an intermediary, such as English as a lingua franca.

You may guess and you can be barking up the wrong tree. When you understand what they mean, maybe they have missed the boat. You can't judge a book by its cover, since sometimes the companies you least expect to have great communications will surprise you, and those who should know better fail to cut the mustard. The world is getting smaller, the internet gives many surely the best of both worlds, and English can be used. Non-native speakers often have a greater technical understanding of English than native speakers, as strange as this may sound. Native speakers may well remember that often idioms should be seen but not heard, for example. If you did not notice, idioms appear in italics!

Hopefully the astute reader gets the point, whether academic, practitioner or curious person who discovered my thesis. The nexus is that many understand something that is understandable, but it may be incomprehensible at the same time. Maybe the reader will put the time in to decipher it, many will not, especially when there are other demands on their attention. Focus on the language, at least.

The university libraries of Malmö, Centria and Tritonia deserve thanks, along with my home municipality

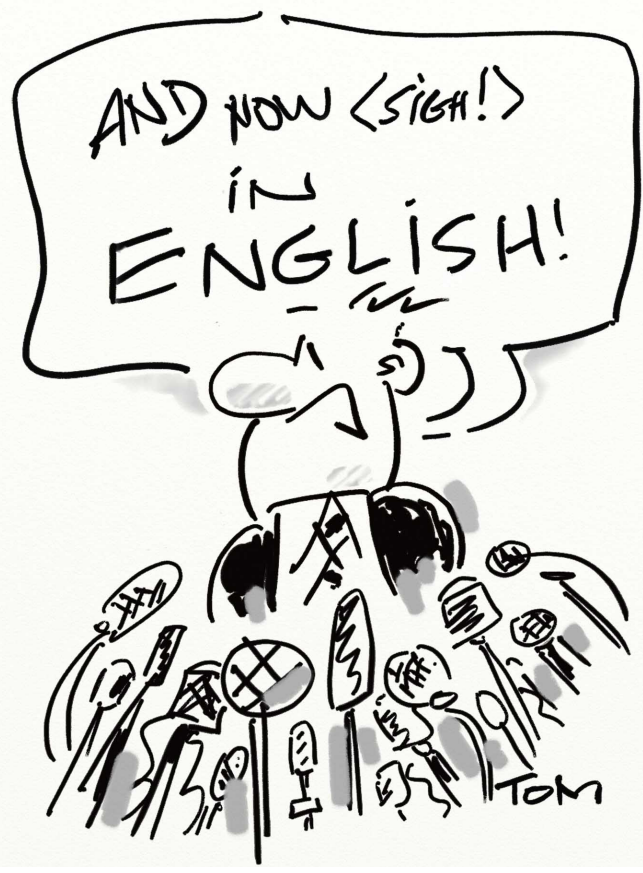
library in Larsmo, for indulging my curiosity and bibliophilic tendencies. My supervisor and other staff at Malmö have been invaluable and I am thankful for their encouragement. I am grateful for the grant by Robert Åke Lindroos stiftelse (foundation) in Finland that supported the development of this thesis.

Sara Ånman deserves repeated thanks for letting me indulge my mid-life crisis, whereby I went 'back to school' to do all that 'university stuff' that I missed out on as a youth, when setting up a company seemed more attractive. Coming back to the world of academia as a middle-aged man, armed with decades of business experience, provided a valuable perspective. Does she know what a tsunami she has unleashed?

Thank you to other academics and professionals, whose counsel, opinion and knowledge I could not and cannot resist taking liberally from as I go along. Like an 'academic jackdaw', there will be a use for that bit of knowledge sometime, somewhere. I just know it, but don't always know 'when'. I should also thank those, in advance, who may have to put up with my questions in the future. This quest is not yet over, I hope! Think of how it can be living with me, so no doubt my wife and daughter need especial thanks, if not a new pair of ear-defenders, for their ongoing support in all its wondrous forms, as without it this thesis and my ongoing studies would not be possible.

If you would like to discuss this further, give an opinion, argue or anything else, do get in touch! E-mail me (darren@ingram.fi) or find me online. 


\section{CONTENTS}

1 INTRODUCTION 1

2 CONTEXT 4

3 LITERATURE REVIEW 9

3.1 The role of PR 12

3.2 A changing world 14

$\begin{array}{lll}3.3 & \text { Cultural and linguistic diversity } & 16\end{array}$

3.4 Deployment Challenges 20

3.5 Socio-cultural considerations 24

i Mass media and communications theory 25

ii Social and behavioural psychology 26

iii Discourse analysis 26

$\begin{array}{ll}\text { iv Neuroscience } & 27\end{array}$

$\begin{array}{lll}\mathrm{V} & \text { Sociology } & 27\end{array}$

3.6 Concluding thoughts 29

4 RESEARCH FRAMEWORK 31

4.1 Research approach 32

4.2 Research paradigm 33

4.3 Discourse analysis $\quad 35$

5 RESEARCH METHODOLOGY 38

5.1 Common research pool 38

5.2 Qualitative study 39

5.3 Quantitative study 41

5.4 Validity 43

6 RESEARCH ANALYSIS $\quad 45$

6.1 Statistical analysis 46

7 DISCUSSION AND CONCLUSION 57

$\begin{array}{ll}\text { REFERENCES } & 65\end{array}$

\section{FIGURES}

FIGURE 1. Factors influencing ELF 12

FIGURE 2. Mean presence of weak verbs per company $\quad 47$

FIGURE 3. Mean presence of filler words per company $\quad 47$

FIGURE 4. Mean presence nominalizations per company 48

FIGURE 5. Mean entity substitutions per company 49 
FIGURE 6. Mean negations per company

FIGURE 7. Mean noun clusters per company

FIGURE 8. Passive voice usage per company

FIGURE 9. Mean modals per company

FIGURE 10. Mean rare words per company

FIGURE 11. Mean extra-long and extra-short sentences per company

FIGURE 12. Mean word count per company

FIGURE 13. Mean words per sentence per company 54

FIGURE 14. Mean readability analyses per company 55

FIGURE 15. Experimental sentiment analysis per company 


\section{INTRODUCTION}

Globalization leaves its mark everywhere, whether good, bad or indifferent, evidenced by change and opportunity. This is noticeable in business and the media, where intermediaries may be eschewed or side-lined in favour of a direct relationship or connection, irrespective of geographic and linguistic barriers.

You don't get a second chance at a first impression. This can be critical for companies seeking to promote their activities, whether they are communicating with the media or a prospective customer. An approach can be examined within seconds. Headlines and introductions are glanced at, eyes ready to dart to the next piece of content in whatever format it may appear in.

$\mathrm{PR}$ is a clear part of media and communications. In the form of a press release it is a message, something that can be remediated into different media formats, as well as being directly read (or viewed, or listened to) by media consumers. It helps shape an impression about an organization, which may in turn be recommunicated in both media (e.g. social media) and non-media (e.g. word-of-mouth) forms. The impression can also lead to a cause-and-action effect, such as encouraging the purchase of a product or the support of an idea or concept.

English is a dominant global language. This can be seen on the internet, with its use as a common, intermediary language. Communications-led activities require good, clear and unambiguous language. This can be even more critical when using English as a Lingua Franca (ELF). Just having a native speaker help shape communications may not be enough, it can be possibly both a help as well as a hindrance!

Research has considered how English is used within business communications, both internally and externally ${ }^{1}$, and there is no shortage of research into linguistics

1 Key reading includes Franceschi (2017), Holtgraves (2013), House (2002), Kankaanranta (2005), Louhiala-Salminen and Kankaanranta (2012), Pickering (2006), Planken (2012), Valentini et al. (2016), and Volistedt (2002). 
and other related disciplines. I believe that consideration has not been sufficiently given to the linguistic structure and stylistic approach of the communication as deployed within PR activities, and I argue that this can impact on audience reception and subsequent activity, particularly when using ELF as an intermediary language.

Louhiala-Salminen and Kankaanranta (2012) are clear that language is not a factor to be forgotten and emphasize, referencing others, that it is an issue that needs to be investigated and it deserves strategic attention from organizations. Even though they have focussed on internal communications, it still has considerable validity concerning external communications.

I suggest that many companies may not be communicating efficiently and effectively when using ELF. My interest has been inspired by prior examination of how companies use PR and communications activities within their export process (Ingram, 2016), as well as an extensive period working as a journalist, dealing with companies from all around the world.

My research suggests a knowledge gap and extant problem with the use of ELF within PR, despite broader research existing in other areas such as internal business communications, that consider how employees interact with others. Articulating and communicating a 'broadcast' message to a much more diverse and 'uncontrollable' audience can be more challenging, and anything that helps improve the chances of this message being received, understood and positively reacted to should be viewed as a good thing. Every message would be framed as part of the usual course of operation (Hallahan, 1999), although consideration may not be necessarily given to the quality of 'tools or material' being deployed and how this impacts on its delivery and subsequent reception.

The aim of this thesis is to examine the linguistic structure used within English language PR messaging by major Finnish companies. I consider that this may have relevance in the broader use of ELF within PR, which may benefit other business areas too. The potential to impact and further influence the communication is desirable. The ability to remove the risk of misunderstanding, 
reputational damage and more that a 'consumer' of the mediated message could experience through a poorly communicated communication should be viewed as being critically important. The core arguments concerning language structure, accessibility and understanding have broader relevance in business, and can also be applied in other media/communications areas such as journalism, social media communications and advertising.

This thesis is exploratory in nature, taking the form of a research-led narrative review (Bryman and Bell, 2011, pp. 110), drawing on quantitative technical analysis of PR communications and assessment of the same data. Narrative interviews with communications personnel feature to detail real-world implementation.

I intend to consider the complexity of language deployed within PR messaging and examine the possibility that comprehension and efficacy could be improved by changes to its linguistic structure. To that aim, two related research questions are central:

How do companies, from a non-English speaking country, formulate their public relations messaging, intended for external publics, using English as a (possibly shared) lingua franca.

Does the structure of deployed language inhibit communication or reduce the possible efficacy of messaging, and if appropriate is there the potential to encourage change through a field-implementable process or framework.

Chapter two provides context into the importance of effective communications and PR activities, particularly when using ELF. This is followed by an extensive review of literature in chapter three, split into several sub-chapters to corral different thoughts and contributing areas together. Chapter four details the research framework, and the research methodologies of the thesis are presented in chapter five. I analyse the research in chapter six before a discussion of the broader findings and conclusions in chapter seven. A list of references and appendices with supporting information then follow. 


\section{CONTEXT}

To effectively communicate, we must realize that we are all different in the way we perceive the world and use this understanding as a guide to our communication with others. (Robbins, 2003)

The internet and social media has changed the common definition or understanding of global public relations. A company does not need to be knowingly seeking international business to be present with such an activity, although should they be purposefully doing then the issue does become acute.

Public relations $(P R)$ is a very broad term. Many who work in the PR industry are confused about what it is, thinking that it is 'simply communications and publicity' (Bruce, 2015). It is no wonder that businesspeople and the public can be misinformed.

The Oxford English Dictionary gives a technical definition of PR as 'the relationship between an organization or an important person and the general public; the occupation of establishing or maintaining a good relationship between an organization or an important person and the general public' (OED Online, 2015). This is achieved by communication, of course, but what is communication? There is no single definition in the Oxford English Dictionary either, with it featuring three distinct categories concerning senses relating to affinity or association, senses relating to the imparting or transmission of something, and senses relating to access ${ }^{2}$ (OED Online, 2017). These individual senses are distinct on one hand, yet they all can have a direct relevance to PR as an art and activity. Social media network connection(s) will no doubt join such a definition in the future.

2 Some of the (paraphrased) definitions are key: 'the fact of having something in common with another person or thing; affinity; congruity', 'the action of communicating something [...] or of giving something to be shared', 'something that is communicated, or in which facts are communicated; a piece of information; a document containing information', '[rhetoric] the device of appearing to consult one's audience or opponent' and 'means of access between two or more persons or places; the fact of being connected by a physical link, or by a practicable route; connection, passage' (lbid.) 
Communication is important and within an organization it differs from other functions since it includes three aspects: a 'performance function' where activities are realised, e.g. writing a press release, a 'management function' where such activities are disposed and aligned, e.g. leading a communications team, and a 'second-order management function' that influences the management behaviour of executives and their peers by providing public opinion and views about the organization (Tench et al., 2017, pp. 118-119).

English is often 'a 'contact language' between persons who share neither a common native tongue nor a common (national) culture, and for whom English is the chosen foreign language of communication (Firth, 1996, p. 240). English is a lingua franca, an international English for business purposes (Bartlett and Johnson, 1998) and has become a term in its own right - English as a Lingua Franca $(E L F)^{3}$.

Estimates vary, but broadly suggest that 375-500 million people may have English as a mother tongue (native speaker/NS), while 1-1.5 billion have it as a secondary language or (non-native speaker/NNS). Other languages are, of course important, and where possible it is preferable to communicate using a shared common NS language. However, English remains, thus far, the most prominent and visible intermediary language and therefore considering how an ELF-based message is structured and mediated is important.

Some might ask whether this is important today, when automated machine translation is only a mouse-click or app-press away. Machine translation has improved dramatically over the past decade and Google has become a key participant in this area with its Google Translate service, serving over 100 language pairs and being used by over half-a-billion users worldwide

${ }^{3}$ Some researchers refer to 'Business English as a Lingua Franca' (BELF) to concentrate on the use of English within a conceptualized globalised business environment. As my research's focus is on outward business communications and how this may impact on public relations activities, both forms may be used interchangeably, unless explicitly stated to the contrary. 
(Google, 2016). As improvements occur, areas of deficiency become more noticeable and acute, so sociolinguistic differences, industry terminologies and a 'feeling' for language can be highlighted with problems that follow (Mundt and Groves, 2016).

Thus, the practitioner heaves a sigh of relief: Brilliant! We can use English and automated translation when necessary - it is the answer to our prayers. Much time and stress can be saved, thanks to this technology. Or, according to Google Translate in Finnish 'Loistava! Voimme käyttää englantia ja automaattista käännöstä tarpeen mukaan - se on vastaus rukouksiin. Tämän tekniikan ansiosta paljon aikaa ja stressiä voidaan säästää'.

In fact, the foregoing is a good example of machine translation. There was only one small error in the Finnish version (a missing 'a'). However, examining the same text through the Bing translation tool produced a quite dissatisfactory translation. Having good, clear language from the start helps. We are human; yet we do not always write or talk in such a simple, structured way.

What about if I wanted to use ELF to communicate the meaning of the following Finnish government press release? 'Tulevaisuuden Valtiopäivät kokoaa kansalaiset, päättäjät ja asiantuntijat torstaina 4. toukokuuta Porvooseen, jossa Suomen itsenäisyyden sadan vuoden yhtäjaksoisen demokratian jatkoksi etsitään uusia muotoja demokratian toteutumiselle ja kansalaisten kuulemiselle' (Valtioneuvosto, 2017). I might use Google Translate, which suggests the following 'The Government of the Future will gather citizens, decision makers and experts on Thursday 4 May in Porvoo, where new forms of democracy and the citizens' consultation will be sought after the continuation of the 100 years of uninterrupted democracy in Finland.'

This wasn't a bad attempt, although when compared to the official 'human' translation some modest comprehension and understanding issues were observed - 'The Parliament of the Future is an event that brings together citizens, decision-makers and experts for a meeting in Porvoo on 4 May to discuss new forms of democratic and civic engagement in continuation to Finland's 100 years 
of democratic government' (Prime Minister's Office Finland, 2017). This illustrates how a practitioner truly concerned with accurate, clear communication in ELF cannot rely on machine translation.

The target audience and use of the communication can determine, in part, the writing style and the language deployed. Complexity matters too. Human readers can be more forgiving and blank out parts of a sentence they do not understand, or seek to find a suitable word they understand in their own language as a bridging mechanism. A machine-translation will carry on regardless and may produce a very strange, impenetrable alternative.

Greater thought and consideration when the originating text is being written, by both NS or NNS alike, is necessary. That is my contention. Clarity and complexity need to be considered in a sensitive way, yet there is a place for unambiguous, precise and focussed language in certain situations. Getting the balance right is the difficult part.

Consider the two translated pairs of text shown previously. The first pair's English language version had a Flesch-Kincaid Grade Level (Stacks, 2011, p. 135) machine-calculated readability score of 6.6. The target for a member of the public is around eight. By contrast, the second translated text scored 19.2. This is quite a difference. As an English NS, the second text did not pose any comprehension problems, even though my 'journalistic mind' would have invariably tweaked a couple of words although they did not really offend or disturb me. Google Translate's output was understandable, even with some nuances lost, yet I wouldn't advise it as a source of professional communication without additional work, even if it was a choice of last resort.

Maybe the source language could be clearer, for the benefit of both NS (who might not have necessarily such a great grasp of their own language), as well as the NNS. It may also make things easier for automatic translation tools and Internet agents that process textual data at the same time - a welcome, but unintended side-effect. 
Academia, PR or writing a letter to the girl or boy of your dreams, it remains the same when it comes to communications. Clarity is a key part of writing or communication style, as 'if your public doesn't understand what you've written, your efforts will have been wasted. But even if your writing is clear, a dull style can put your readers to sleep, and your message still won't get across' (Newsom and Haynes, 2008, p. 110). If you make your text clear, and understandable, it will make your communications processes easier and lead to greater reception, particularly by NNSs (Mauranen, 2006). ELF has its place, yet 'the introduction of (often quite bad) English as the business lingua franca is only a partial solution to the problem; often the majority of employees and even more customers and other stakeholders are not able to communicate in English,' noted (Verčič et al., 2015).

How companies deploy their messaging using ELF can be relevant, especially if the use of language is overly complex, negative or possibly unintentionally misleading or inaccurate due to cultural grounds and unfamiliarity. This may pose a problem, or add an imbalance, to any public relations activities that use ELF to reach their publics. This indicates that an often-subconscious translation or remediation of messaging is made from the ELF-originated message to the recipient's strongest and most natural language and any further outward communication may go through a similar process.

In other words, if the message being transmitted is 'less clear' after it has been translated to ELF, its reception and translation from ELF to the recipient's own language may be similarly affected; and this is even before the message may be diluted or miss-communicated if recommunicated. Anything that can make the message clearer, more understandable, or less prone to miscommunication must be a good thing. Especially concerning international communications and contact.

This issue is even more acute today, as companies are increasingly (and additionally) communicating direct with their publics instead of going through 'gatekeepers', such as a professional journalist, who traditionally would remediate such PR-inspired messaging after passing it through a 'filter' with some processing and enhancement before delivering it to the reader, possibly written in a shared language. 


\section{LITERATURE REVIEW}

Globalization and the breaking down of media and communications barriers by the internet and social media has brought forth many benefits to businesses. It has also posed communications challenges, particularly since it is tied intimately to consumer/buyer behaviour (Albaum and Duerr, 2011, p. 778), which is determined by many effects, including underlying cultural differences related to perceptual understanding and acceptance, e.g. hard-sell versus soft-sell (Okazaki et al., 2010), messaging content (Lin, 1993), host and recipient organizational differences (Balmer and Wilson, 2001), and even the inherent trust given to the media-at-large and broader social trust constraints (Braun, 2004).

According to Vuckovic (2008), there are five overlapping factors that impact our ability to competently communicate inter-culturally - culture, perceptions, roles and identities, communications style, and personality - and these can have bidirectional implications. Singer (1987) emphasizes that there is an important connection between perception, a selective method partially determined by culture whereby an individual permits certain information through their 'perceptual screen', and communications style, both the method of how a communication is formed and distributed as well as the deeper connection it establishes (Samovar et al., 2013, ch. 8). This connection can be influenced by the careful use of deployed, understandable language within messaging that may lead to greater reception, acceptance and possibly a positive reaction thereafter.

I argue that despite international PR activities being increasingly commonplace and visible, whether intentionally or not, many PR practitioners and companies are not paying sufficient attention to the deeper construction of their messaging and this can have a detrimental effect, especially when using English as a common language.

The evolving solution to the challenge of global communication has been to use English, the lingua franca of business. The growing international dominance of English language media and culture, aided by the 'shrinking' of the world by the 
Internet, helps further establish English as a de facto lingua franca, even though this fact and the underlying motivation for it is not without critique (O'Regan, 2014). This makes it even more important to study its utilization in all areas of business communication. As Charles (2007) concludes 'language does matter in global operations', 'language matters are important for companies' and that '...heightening awareness of communicative and cultural diversity and working on ways to increase mutual understanding of the Englishes (or other shared languages) used globally [...] is of vital importance' (Charles, 2007, p. 279). The growing acceptance of ELF brings many advantages to global business activities whilst, at the same time, adding risks too.

ELF has been described as an 'enabler of communications' (Louhiala-Salminen and Kankaanranta, 2012) and a 'language always in translation' (Pennycook, 2008), so its importance as a communication tool should not be overlooked. However, it is just as important to examine the challenges uncovered through the study of the use of ELF.

Although the broader areas of media studies, public relations, cultural studies and psychology touch upon the challenges of international communication and language, specific research into the effects of language on global business messaging is mostly absent. Examining literature relevant to this focus is thus challenging on one hand, whilst beneficial on the other as it latterly supports and informs this work. I am seeking to focus my evaluation of literature accordingly on key elements that influence this specific research area, whilst highlighting possible complementary areas of interdisciplinary research for the future.

Although my focus is on the external PR activities of a company, clearly many of the same issues raised may have a similar impact on its internal, global communications when suitably abstracted. As White et al. (2010) maintain, effective internal communication can 'enhance corporate reputation and credibility' as employees are viewed as 'particularly credible sources by external stakeholders' and 'have a powerful influence on organizational success'. Intriguing as this is, it falls beyond the scope of this work. 
The global use of ELF for business communications is an accomplished fact of our modern world. In this chapter, it is important to examine the factors that have led to the increasing utilization of ELF in PR, which include the basic role of PR as a business function, the changing nature of global communications, and the diversity of culture and language that a successful global business must navigate.

More importantly, and less well-understood so far, are other factors that can shape the use of ELF and influence the success of communications attempts. There are both practical linguistic deployment factors that direct the use of ELF and broader socio-cultural and even perhaps even biological elements that affect ELF communications. At the same time, each factor can influence another factor, and be influenced by ELF in return. These can be anticipated by examining the theoretical perspectives of various academic disciplines. Together (see figure 1), these lay the groundwork for a research examination of the use of ELF in PR, and for recommendations for practitioners to enhance the success of their communications activities.

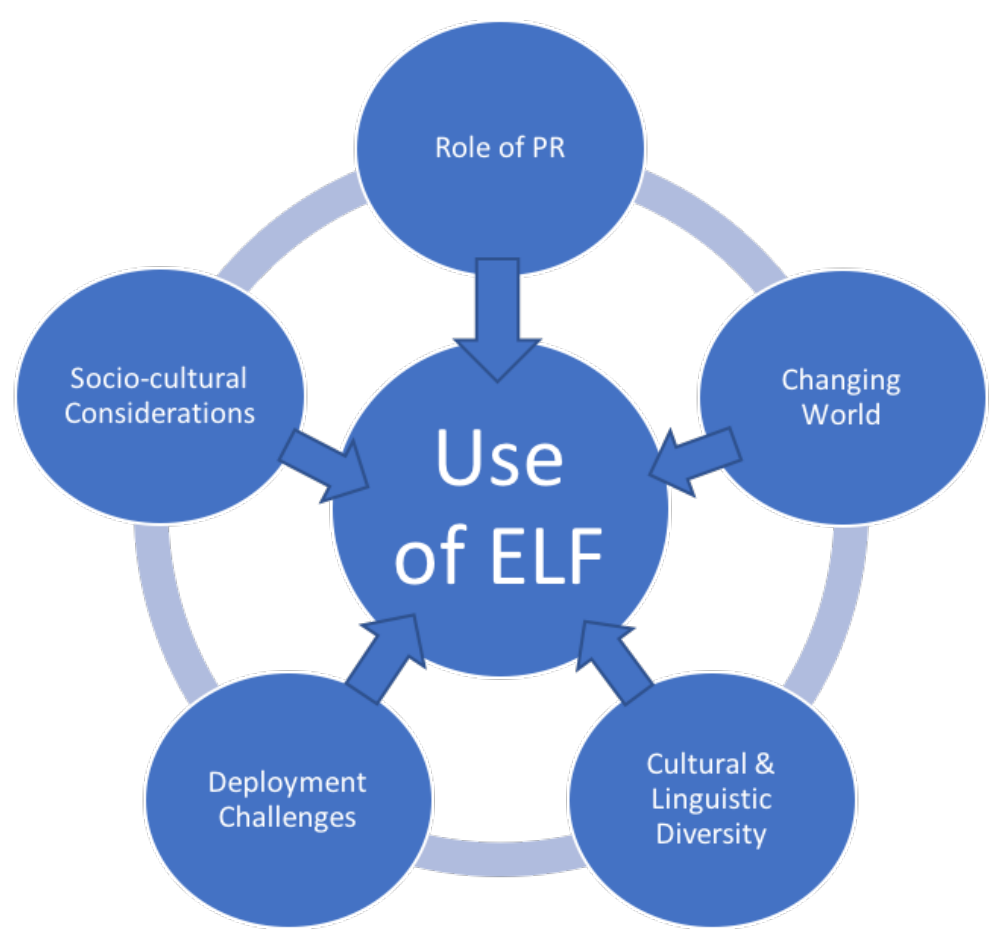

FIGURE 1. Factors influencing ELF 


\subsection{The role of PR}

ELF as used in PR is first and foremost a form of communication. As with any form, when looking at how communicators communicate, it is essential to briefly look at how they describe their own activities. The Chartered Institute of Public Relations, a 'leading representative body for the PR profession and industry in Europe' (Chartered Institute of Public Relations, Undated), defines PR as being 'about reputation - the result of what you do, what you say and what others say about you. [...] the discipline which looks after reputation, with the aim of earning understanding and support and influencing opinion and behaviour. It is the planned and sustained effort to establish and maintain goodwill and mutual understanding between an organisation and its publics' (ibid.).

Use of the plural form of public is purposeful. There is more to PR than (trying to get) media coverage that can persuade or influence customers to buy a product or service, even if it is an important part of it. PR is not a synonym for publicity. Other publics, excluding possible or actual consumers, can include shareholders, government departments, employees, external pressure groups and even industry partners, peers and rivals (Blythe, 2006, p. 137; Burnett and Moriarty, 1998, p. 347; Pickton and Broderick, 2005, p. 559).

Publics can consume or be receptive to activities other than 'news publicity' that may be mediated directly or indirectly through consumption, such as sponsorship, product placement, lobbying, reputation management and advocacy (Blythe, 2006, p. 145; Kotler et al., 2009, p. 744; Kotler and Armstrong, 2010, p. 472).

Simmons noted 'When a boy meets a girl he tells her how lovely she looks, how much she means to him, and how much he loves her, that's sales promotion. If, instead, he impresses on her how wonderful he is, that's advertising. But if the girl agrees to go out with him because she's heard from others how great he is, that's public relations' (Simmons, 1991, pp. 133-135).

Maybe that date was influenced by other matters, such as reputation. As you communicate a reputation is formed, which can open doors and close them just as 
fast. Benjamin Franklin had some humorous but wise words hundreds of years ago that remain valid today: 'Glass, china, and reputation are easily cracked, and never well mended' (Ford, 1889, p. 201).

A company's reputation 'represents organizational past and present performance and portrays the ability to deliver reliable results to various stakeholders' and 'illustrates the perceptual track record of organizations' (Gibson et al., 2006). Reputation is formed inter alia by how communication is made to one's publics and is far more than just sending out a press release. Reputation is arguably the single most-valuable asset an organization can possess (lbid.), the benefits of which are like the value of relationships with various publics, and although they are separate entities, PR can improve relationships, adds value to the organization and that, in turn, will influence reputation (Grunig and Hung-Baesecke, 2015).

The use of a common language reduces linguistic and cultural distance in general and this can be extrapolated whereby ELF is used (or any other common language). This can have impact on many other areas of the organization that may be touched upon by PR or related activities, such as sales (or even investments into the company). Cuypers et al. (2015) examined cross-border acquisitions and noted 'the effective use of a lingua franca is affected by the native tongues and cultures of the parties' and discovered that 'acquirers take lower equity stakes in foreign targets when linguistic distance and differences in lingua franca proficiency between them are high, and take higher stakes when the combined lingua franca proficiency of the parties is high'.

Relationship and relationship building/management should be priorities for companies, as individuals tend to strategize when demonstrating commitment (to an idea, company, etcetera), namely they listen to the concerns of the relational partner, they demonstrate empathy, they spend time with the other, they use language that is understandable and they remain open to change (Adler and Proctor II, 2015; Cuypers et al., 2015; Grunig and Hung-Baesecke, 2015).

The use of ELF presents unique challenges in this area, since building relationships between users who do not share a common language opens much 
more potential for falling short on these critical areas. These are all aided via communications-based activities and the transmission of a message through a medium.

A press release ${ }^{4}$ is a common way for a company to announce its news and seek publicity within the mass media, in the past necessitating going through a 'gatekeeper' such as a journalist who may, in turn, write an article (or produce something in another media format). Today the gatekeeper's role is less exclusive due to the internet-at-large. The press release is still be at the heart of a company's messaging, sent to external media, published on its own website and social media networks, repurposed for use within internal communications and external customer publications and forming part of (for some) a regulatory requirement. Its format and utility may have changed, its once-reverential tone diminished and its death stated in error many times (Owen, 2017), but it remains at-heart an important and even a good tool for messaging design, even if it is not used in the once-traditional way.

A relationship between PR representative and journalist can make the news dissemination process easier, but there are two key factors that can ultimately determine whether a journalist 'bites' on the story: news value/content, i.e. impact and textual properties of the 'media product' used to relay this (Sumner et al., 2016; Superceanu, 2011), commonly known as the press release. Study has been made into roles of informativity and linguistic intertexuality within a press release, but not as a piece of external messaging that has been either authored with, or is intended for, reception by those who require use ELF.

\subsection{A changing world}

ELF has developed and been established at least in part because of the increasing and changing communication demands of the modern business environment. Companies communicate to their publics, increasingly bypassing intermediaries as discussed in the previous sub-chapter, through social media,

${ }^{4}$ McLaren and Gurău (2005) offer a good overview of the genre of a corporate press release, explaining its construction and its linguistic elements. 
business-to-consumer marketing (B2C) contact, internet presence, etcetera. This can be clearly a mass media activity itself, as the message's originator is also the publisher.

It is quite possible that the message may lose an element of objectivity as well as linguistic interpretation and smoothing that a gatekeeper may have provided. Some larger companies may employ former media professionals to assist in these tasks, although it still may lack a certain independence, overall quality level and linguistic styling. Some of my research has noted PR messaging that has allegedly been handled by former 'top journalists', which had acceptable grammar and reasonable content, but it did fall on the grounds of ELF comprehension.

It should be noted that there is a wider choice of media available today, with reduced barriers to entry. Everybody can be a publisher. Different media forms present different challenges, yet ELF-based messaging adds an even-greater set of issues that may be media-dependent which, in turn, may affect audience consumption (Palmer-Silveira et al., 2007, pp. 12-13): with a gatekeeper involved, some of these issues could be ameliorated.

Globalization has significantly increased interaction among organizations and publics, necessitating the need for international PR. Consideration of such an activity may be necessary in many cases too, even if the organization does not have any international activity, since very few organizations operate in a true local vacuum. Technology can be a blessing and a curse. Sriramesh (2009) noted that 'the expansion of communication technology has increased the dissemination of information about products, services, and life styles around much of the world, thereby creating a global demand for these products'. Johanson and Vahlne (1977) warned decades ago that a 'lack of knowledge due to differences between countries regarding, for example, language and culture, is an important obstacle to decision making connected with the development of international operations': It is equally relevant today.

This globalization and greater use of foreign languages (of any kind) can even be 'injurious' to your health, or at least place additional stresses on vocal cords due to 
pitch changes, noted a recent research-based doctoral dissertation (Järvinen, 2017). As an afterthought, having a clear-to-understand deployment of language, but particularly BELF, may stop people subconsciously reading out aloud something that may appear unfamiliar or difficult to comprehend, giving slight relief to both brain and vocal cords alike!

\subsection{Cultural and linguistic diversity}

Language is an important topic for businesses to master. Because it enables individuals and companies to communicate, the development and use of language and communications through which it occurs is critical (Charles, 2007). Clear communication is acknowledged to be necessary for business, of which PRrelated activities are a central tool. Research into disciplines such as international marketing, exporting and management over decades has emphasised the importance of language considerations when selecting foreign markets and selling to overseas customers (Brannen et al., 2016, p. 144).

The linguistic diversity of message recipients has led to considerations that directly impact the use of ELF. This language and its underlying message is also shaped by contributing cultures and languages possessed by the writer(s) and other collaborators and obviously will be similarly reflected by recipients too (LouhialaSalminen and Charles, 2007, pp. 31-33; Palmer-Silveira et al., 2007, p. 11).

Language is a static system, built around competences such as rules of linguistic structure and theories including language expectancy (Dillard and Pfau, 2002, pp. 119-133) that form a broader part of communications theory (Cornelissen, 2004 , pp. 16-18). Language also plays an important part of the communications process, both in the formulation of a message and how it is received. Yet language is a lot more than mere linguistics.

One interesting difference, observed between the understanding of ELF and the more specialized notion of BELF, is that BELF researchers see communications skills as crucial, whereas ELF researchers see linguistic skills as the crucial factor in communication (Charles, 2007). A small difference, you may argue, but it can 
depend perhaps on what end of the telescope you are looking through and what you are looking at. This is where the problem can lie, since language is a tool to aid communication, and every person will react differently to it through different stimuli. Louhiala-Salminen and Kankaanranta (2012) raised a valuable distinction between ELF and BELF, with BELF portrayed 'as 'simplified English' since typically it did not contain complicated phraseology, idiomatic expressions, or complex sentence structures'. Clarity and readability could be key, since 'grammatical correctness was secondary and was not perceived nearly as important for successful communication as specialized vocabulary' (Ibid., p. 13).

For this thesis, there is less of a fixed division between ELF and BELF, since both forms can be relevant. ELF and BELF should not be confused with common corporate language $(\mathrm{CCL})$, an internally focussed language that may be used by an organization, which may be English but not automatically so (Piekkari et al., 2014 , p. 8) that is not without its share of potential pitfalls as well as benefits (Welch et al., 2001).

Even companies that operate in the same sector may not share the same ELF terminology, and cultural/societal differences can adversely change their communications style and approach, leading to greater interpretational and reactional issues for messaging recipients. In a study, Skorczynska and CarrióPastor (2015) compared the use of 'general meaning keywords' in press releases from energy companies in Britain and Spain, noting that they varied substantially from each other, reflecting 'not only the different communicative strategies at work, but also the distinct social and cultural contexts from which they undertake their global operations'. In this sense, the press releases studied seem to address issues which are important to their societal collectivities.

Regarding the use of language within global communication, Charles (2007, pp. 270-277) suggests the relevance of five broad statements: informal, oral communication deserves to be considered of paramount importance; language is a power-wielding instrument; language affects perceptions of oneself and others; language unites people and organizations - but also divides them; and BELF is not a 'cultureless' language. These elements can have varying relevance within 
PR activities, dependent on individual tasks and processes. They may also be extant within other business processes that impact on, or are impacted by, this PR activity, both internally at the originating company (and related stakeholders) and externally within the recipient company/companies, industry-at-large, other stakeholders and impacted parties.

While general business communication within a culture has obvious importance, and presents key challenges, inter-cultural communication, such as is required by global business activities, is exponentially more fraught with risk, due to the increased potential for miscommunication across cultures. Some communication mistakes may appear to be humorous at first sight, yet costly to rectify, such as using a potentially misleading word. A U.S. manufacturer discovered this by introducing a product ('Pet milk') into French-speaking markets without understanding that 'pet' in French means 'to break wind'.

Other challenges may be more analytical and focused, based around the differences between low- and high-context cultures, where language can varyingly express thoughts, feelings and ideas to different levels (Adler and Proctor II, 2015, pp. 177-178). The challenges of inter-cultural communication are about more than just translation and localisation. Adding to the complexity, there can even be significant differences between the sexes who share the same language and culture (Ibid., p. 182).

Even though English is a key, shared language of global communication, it is important to note that for most users of English - roughly three-quarters - it remains a foreign language (Seidlhofer, 2005, p. 339). Despite its shared nature and positioning as an intermediary language, English linguistic competency and familiarity between users can vary tremendously, along with comprehension and cultural understanding differences. Vocabulary and structure also matter (HsuehChao and Nation, 2000; Johnson, 1981; Perfetti, 2007, pp. 359-360; Steffensen et al., 1979) and these impact everybody, but particularly NNSs who use ELF.

This is even before consideration is given to other problems that can exist, such as misunderstandings of communications sent through various media types, which is 
outside the scope of this thesis. For example, even native speakers of a shared language can have difficulties, such as failing to identify and associate with emotion in emails, and differences can exist on grounds such as intimacy and gender as well (Kato et al., 2010).

The subject of readability and the use of readability formulas, which is apposite within my research, is not considered in detail within this literature review, since its role is that of a tool. In this situation the selection of a specific tool over another may be less relevant if it is used consistently and its output considered in context (Redish and Selzer, 1985; Selzer, 1981). I am aware that there are many discussions about the different formula and many good articles on the subject, including by way of example Chall (1947), Fry (1968; 1990), McLaughlin (1969) and Meade and Smith (1991), but these mostly are written from a linguistic and/or pedagogical perspective.

There is also justified concern about the blind implementation of readability formulae and observance of results, based around the narrow conceptual focus on readability text analysis (Stubbs, 1990, p. 314), which I can agree with. In broader research, I would consider additional, extra measurement techniques that evaluate audience comprehension (Mosenthal and Kirsch, 1998; Stevens et al., 1992), since my broader hypothesis is that many elements jointly contribute to the issue. I maintain that readability analysis is a useful field-deployable tool to aid practitioners, but not the only weapon in their arsenal.

As a footnote, one concern noted by Verčič et al. (2015) was whether increasing sensitivity to other cultures and languages may result in the danger of decentering one's own key values, making managing communication even more complicated. As valid as it is, I do not consider this to be an issue when using BELF, although it may be relevant should BELF be advocated at the expense of local language communications. 


\subsection{Deployment Challenges}

Due to the growing acknowledgment of ELF as a practical requirement of global success, NNSes are 'habitually engaging in natural spontaneous discourse in English even at lower levels of competence, and their objectives are not so much emulating native speaker behaviour as accomplishing real world tasks' (Christiansen, 2016, p. 95). Their formal linguistic competence is less relevant to their actual communicative performance in many, if not most, situations.

Getting the language right and more importantly understood by one's publics is thus more important than ever before and those shaping and originating the communications message have the onus of responsibility for ensuring it is as understandable, unambiguous and (from their perspective) as attractive and likely to attract engagement as possible. A degree of simplification and clarity within the text should be obligatory (McWhorter, 2007), as part of the shaping and message framing process.

This obligation can be considered even more important when one considers the communications hurdles with a 'filter' of using ELF, such as differing levels of comprehension and inter-cultural complexities (Franceschi, 2017). Further, differences in translation encoding (origination) and decoding (reading) can be obstacles. For example, even if we possess foreign language competency, subconsciously we translate what we understand back to our own native language as part of the comprehension process, adding to the risk of miscomprehension.

There is a risk, however, that creating ELF-friendly text can be 'dumbing down' a NS-level text, if care is not taken, since 'ELF will thus become gradually more lingua franca and arguably less English with ordinary users becoming more sophisticated at mixing and matching languages from their linguistic repertoires to communicate in the globalized context that the internet has brought into their lives' (Christiansen, 2016, p. 95). This may be avoided through the careful, professional and considered deployment of language by communications professionals thinking globally, although this obviously presents significant challenges. 
Concern has been raised that ELF is even a 'threat' to national languages and multilingualism (Apeltauer and Shaw, 1993; Decke-Cornill, 2002), but this can be countered by viewing ELF as a 'language for communication' rather than a 'language for identification' as House suggests (House, 2003a) ${ }^{5}$. Seidlhofer (2007, pp. 148-149) stated that 'sociolinguistic research suggests that if - and this is a vital condition - English is appropriated by its users in such a way as to serve its unique function as ELF, it will not constitute a threat to other languages but leave other languages intact, precisely because of its delimited role'.

Where material is translated from English (in any situation) there can be a risk that it is not passed through a 'cultural filter' that may preserve 'local communicative conventions' (House, 2003b, pp. 168-169), leading to a 'shift from cross-cultural difference to similarity in textual norms and text construction' by reflecting Anglophone norms. I argue that this is less relevant for PR messaging, although clearly a quality localization (Chaffey et al., 2006, p. 317) can be more significant for some marketing, publishing and other activities and should not be overlooked. Cultural issues can create impediments for ELF usage for both NS and NNS user alike (Maclean, 2006), yet users are counselled not to cut linguistic complexity at the cost of communications efficacy.

A traditional piece of PR messaging should be more informative, matter-of-fact and clearly framed (Hallahan, 1999), but it may be (should be) devoid of overt marketing language to differentiate it from a brochure or advertisement (Catenaccio, 2008). Bluntly put, if it is constructed well, a journalist could quickly edit it and present it for publication, irrespective of the ethical and presentational issues that may occur (Maat, 2007; Maat, 2008; Maat and de Jong, 2013; Raeymaeckers et al., 2012, p. 148).

${ }^{5}$ Discussion of this threat is more suited to a politics or linguistic thesis, but Phillipson (2001) produced an interesting article that considered the role of English within globalisation, reasons for its dominance, and the need for conceptual clarification in analysing English worldwide, which remains valid today. Two trends were discussed that featured 'competing language policy paradigms that situate English in broader economic, political and cultural facets of globalization', namely the Diffusion of English paradigm, and the Ecology of Languages paradigm (Ibid.). 
On the other hand, some researchers believe that ELF can exist at the same time as a growing body of plurilingualism, meaning that NNSs may deploy languages other than English too, leading to a further conundrum for some (Christiansen, 2016, p. 88). This affects, or benefits, particularly the young, who are inevitably experiencing plurilingualism much more than preceding generations, whether through family ties (e.g. my own daughter who has had to simultaneously learn three languages from birth), leisure activities like gaming, social media and Internet use, or educational pursuits.

Accuracy, or the lack thereof, and the general understanding of any message being communicated can be key. Irrespective of the subject area, common errors in reporting and messaging can be ascribed to both communications professional and layperson alike, with risks including omission of critical information, misquoting and missing contextual information (Brechman et al., 2009). Certainly, this risk is heightened when communication is made between NSSes using ELF, so the nature of the language deployed can be at the very least a mitigating factor.

When using ELF, there are challenges in order not to cause 'intelligibility problems' between the sender and receiver of a message ${ }^{6}$ which could otherwise inhibit the message from being delivered accurately and eliciting the desired positive reaction (Pickering, 2006). For those who use ELF as their shared language and intermediary layer, these challenges can exist in both written and oral communications, the latter exemplified by accent or dialect usage, although this thesis concentrates on the former form.

Ironically, a degree of intelligibility and comprehensibility that may exist in NNS interlanguage talk can be side-lined or reduced when a NS is at one end of the chain. Evidence suggests that 'ELF interlocutors engage in communication strategies and accommodation processes that are unique to this context and may conflict with the ways which NSs typically negotiate understanding' (Pickering, 2006, pp. 227-228). Thus, usage of ELF 'gives an illusion that by controlling the national language diversity the transfer of meaning becomes relatively

${ }^{6}$ In this context, 'message' can refer to either an entire communication exchange or a component part of the broader exchange. 
unproblematic' (Brannen et al., 2016, p. 140). This may lead some NNS communicators to be led into a false sense of security, since many NNSs do communicate successfully (albeit this term is subjective) despite 'anomalies and infelicities in their English as recognised by native-speaker assessments' (Firth, 1996, p. 286).

Where the communication is otherwise comprehensible, even with a modicum of difficulty, it is quite possible that a NS will 'ignore' or overlook the issue if the core is (easily) understood, particularly if the message is otherwise engaging, of interest and focussed to the recipient. That said, in a stressful environment, with a lot of competing messages vying for attention, the tendency of recipients to ignore the more difficult-to-interpret messages or to fail to appreciate their significance can be understood, and this tendency obviously can create risks and missed opportunities for the ELF user trying to communicate with a NS. Even within NS-to-NS situations can these issues be extant due to poor communications style and articulation (Bensing et al., 2003; Redmond and Bunyi, 1993), although those difficulties are beyond the scope of this work.

The efforts involved by the NNS to use ELF can often be great, especially when working with 'artistic' communications such as PR messaging or sales-related activities which are arguably 'hard work' in terms of communicating a message (Charles, 2007). This type of communication is more complex, involved and longer-lasting than ordering food at a tourist destination, for example.

Sometimes ELF users are well-motivated to do the 'hard work' involved in overcoming potential problems with message reception and comprehensibility (lbid., p. 265; Nikko, 2009, p. 38), although it may be unwise for senders to assume that this is consistently applied particularly when, for example, senders are trying to grab the recipient's attention in a flood of other messages. It was also suggested that a shared business background and purpose, irrespective of a diverse background, may increase this motivation and outcome (Nikko, 2009, p. 265). Volistedt (2002) noted that BELF competencies can even be enhanced by the diversity within cultural backgrounds as this reflects on language deployed and similarly understood. 
Of course, understanding a recipient's responses as mediated through ELF can be similarly onerous, whether the correspondent is a NS or NNS. Since NSes may have less than adequate communications skills too (Charles, 2007), neither NS nor NNS may deserve the blame for communications shortcomings. Rather, both parties communicate best when they agree that it is desirable to minimise possible problems and misunderstandings through a beneficial solution.

A lot of the research regarding ELF and business communication focuses on verbal communications in meetings, submitting contents to discourse and cultural analysis $^{7}$. The success of this type of spontaneous verbal communication obviously varies greatly depending on proficiency and fluency. In contrast, my research focuses more on the written word; it can be argued that since a written message can be refined over time before publication, perhaps with cooperation and discussion with others, proficiency shortcomings may be better camouflaged.

\subsection{Socio-cultural considerations}

When considering the broader role of communicating using ELF, attention should be given to other disciplines and their various theories that may have a relevant impact, to create as inclusive an explanation and set of recommendations as possible. In this context, I call them socio-cultural considerations to reflect their broader, inter-disciplinary contribution. These span mass media and communications theory, social and behavioural psychology, neuroscience, and sociology, among others. Elements of these theoretical approaches may contribute to understanding and practice in different, and quite possibly unmeasurable levels. It is important here to briefly examine some likely contributors.

7 For example, one analysis of Swedish and Finnish-language speakers in a meeting using BELF noted Swedish speakers were characterised as being more vocal and 'talkative' compared to the Finnish, yet word counts were roughly comparable. This was explained as Swedish-speakers enhancing communications between partners but saying less about the issue at hand (a wordy, talkative discourse), whereas the Finnish were more linear, arguing and communicating in a monologic, less interactive style (Louhiala-Salminen and Charles, 2007, p. 277). In this work on verbal ELF-usage, comparison was not knowingly made about linguistic proficiency and other measurable elements. 


\section{i Mass media and communications theory}

Media influence theory, for one, interacts with PR communications ideas by emphasizing that the force from a given media message may change or reinforce a behaviour or belief (Shrum, 2009). Speculatively, one can envision that the use of language such as ELF can assist (or impair) this influence (ibid.).

Another media theory, that of the 'hypodermic needle effect' (Williams, 2003, p. 171), may be also examined in the context of ELF, and the belief that media messages are received in a uniform way by an audience that leads to immediate and direct responses that are triggered by such stimuli. Undue weight should not be placed, however, on this effect as it does not consider the influences that intervene between the media and the opinions and attitudes people hold, along with other interpretational issues (Ibid., pp. 171-172).

The perspective of mass media studies can help to foster understanding of how mass media behaviour and reception play into this as well, particularly regarding accuracy. Complaints and concerns about media accuracy are not new. Brechman et al. (2009) made a focussed study into lay press reporting of a serious, technical subject that was quite noticeable in this regard, highlighting issues regarding factual reporting and accuracy, but also explicitly suggesting that the press release $^{8}$ may 'well be a source of distortion in [the] communication' too. Obviously, these distortions in accuracy on both sides of the communications links are relevant and applicable to ELF use. Furthermore, these effects could clearly be exacerbated with changes to the media distribution landscape, such as the increasing role of social media, a growing informality of behaviour and communication style, and the removal of the former gatekeeper acting as de facto interpreter and storyteller. These are concepts drawn from the field of media studies.

More broadly, communications theory can inform this discussion of business use of ELF. Communications accommodation theory (Giles, 2016), for example,

\footnotetext{
${ }^{8}$ Here it is fair to extrapolate 'press release' to read any form of material designed
} for communication to a company's publics. 
describes the ways that individuals accommodate and accentuate their differences by altering their communications style. Understanding and applying this theory has obvious benefits to the study of BELF, as business language users who interact through social media and collaborative/sharing media using ELF may well utilize positive accommodations and thereby create a relevant by-product of clear, understandable communications. This could also negate the real risk of the theoretical 'boomerang effect' (Ross and Nightingale, 2003, p. 25) and other damaging communications mistakes or misunderstandings.

\section{ii Social and behavioural psychology}

Many psychological theories may influence our grasp of ELF communication within PR. Such theories include positive emotion (broaden and build theory), serial position effect theory, social identity theory (Moingeon and Soenen, 2002, pp. 5254), and politeness theory (Holtgraves, 2013, p. 39).

Determining exactly what influenced a given decision in response to a certain message can be difficult - a respondent may claim something from their conscious mind that is entirely different to their sub-conscious reality. These psychological mechanisms affect human behaviour and human relationships, and therefore undoubtedly must be considered when utilizing business communications.

The press release and its deployed language can have a psychological effect (Davis et al., 2006; Davis et al., 2012), although this may rely on a greater nuanced understanding of language and be of less immediate relevance to BELF. It falls outside the scope of this thesis, despite having some relevancies and need for consideration within a broader global perspective.

\section{iii Discourse analysis}

Research into sociolinguistics, pragmatics and even cognitive psychology may have relevance, noted Fairclough (2001, pp. 7-10), contributing to the necessity for conversation and discourse analysis. Fairclough notes the significant role language has in society, and how there can be intertwined relationships between 
language and power and language and ideology (Ibid., pp. 14-15), and this position cannot be overlooked even though semantic differences may exist between his key assertions and their real-world application in this specific case under most circumstances. Fairclough has a lot to say about different elements of language and power that may have a bearing, if exploited, within PR, such as power in cross-cultural encounters and 'hidden power' (Ibid., pp. 40-46) that could be exploited in certain situations within PR activities, although the practicality and possible effects is outside the scope of this thesis.

With the change in the media landscape and a more direct connection being often formed between message originator and message consumer, many beneficial situations may be extant, which may come with a corresponding burden of regulation, ethics and behavioural expectations.

\section{iv Neuroscience}

Language is at heart a biological function of the human brain, and thus even neuroscience may be relevant to our understanding of business communication (Baird et al., 2014; Knickerbocker et al., 2015; Pennebaker et al., 2003; Stanton et al., 2016). Much research is ongoing within neuromarketing and neurosciences and certainly this is an area that PR practitioners should not ignore (McKie and Heath, 2016). Some attempts have been made to identify what may influence us and how we react, yet so far, the human brain is not fully understood! The language deployed can trigger off certain responses that lead to an entire chain of events.

\section{$v \quad$ Sociology}

It has been argued that $P R$ is frequently studied from a management perspective, whilst ignoring its social phenomenon, since it has an important role to play in developing or destroying a company's 'licence to operate', impacting throughout the societal, organizational and individual levels (Ihlen et al., 2009). One intention of communicating with publics is to form a connection and then see a motive realised (whether the motive is to sell a product, pass information or something 
else less relevant to this discussion). Thus, sociological theory may also inform our understanding of PR communications. If a sociologically constructivist approach is taken within PR activity, accepting that this can influence matters through a dynamic social reality of contribution and reflection, as argued by Bentele (1997), you can observe an ongoing process of de- and reconstruction of ideas, values, etcetera, of which PR-inspired communications and advocacy plays a role in the bigger picture.

Communications are inspired by, complemented with, and comprehended through the help of social constructivist theory, whereby development is socially situated and interaction with others contributes to the construction of knowledge. Three key elements from social constructivist theory can guide research: meaning is said to arise from social processes in which individuals contribute; knowledge is created and shared through language use, and taken-for-granted ways of understanding should be challenged (Charles, 2007, p. 267).

A multiplicity of institutions creates networks of diverse stakeholders, both direct and indirect, to communicating companies, whose needs and input may also need to be considered (Akaka et al., 2013, p. 11), and these networks create a complexity of context that it influenced 'not only by the embeddedness of social networks (e.g. local or national) but also by the multiplicity of institutions (social structures) within a given ecosystem' (Ibid., p. 12; Sewell Jr, 1992). Consequently, good use of BELF would reduce misinterpretation, perhaps based on cultural, educational or other grounds, increase 'buy-in' to a message and be more 'shareable' by whatever medium, since we are more likely to share, espouse, advocate and discuss things that we understand, approve of or have an interest in by use of a positive, engaging tone. On the flipside, any goodwill may be lost by misunderstanding and then it is easier to adversely communicate something we disagree with.

This brief survey of the relevance of a broad range of academic theories to the subject at hand does not even begin to scratch the surface. Other apposite theories could relate to the digital sciences, including complexity theory, sentiment analysis and even automated/machine learning assistive aids. Clearly not 
everything can be considered in this thesis and many disciplinary approaches may be contributory in a mass, but less supportive as isolated key elements.

\subsection{Concluding thoughts}

When it is time to put all the pieces together, clearly PR shares many similarities with its marketing and advertising cousins. It can be, if properly deployed, a complex and integral being, forming part of a global service ecosystem. It is embedded within social networks and many partner institutions, utilising the '(co)creation of value influences and is influenced by the enactment of practices and the integration of resource through various levels (micro, meso, and macro) of interaction and institution' (Akaka et al., 2013).

This complexity is helping narrow the once-greater perceived differences between international and domestic marketing and other communications-based activities at the same time. Issues, however, such as cross-country and cultural differences remain, and (exporting) companies must transition from being 'simple suppliers' to 'advocates of their products' (Ryans Jr et al., 2003) that require greater integrated internationalisation and management strategies. Companies must also balance and address the standardized or custom/adapted product/service offering, as part of the marketing mix, with the communications-related issues that befall it (Nakata and Huang, 2005; Ryans Jr et al., 2003).

It appears there is a specific knowledge gap in the role language (ELF in particular) may play with regards to PR and communications messaging. Much research has looked at elements such as the role language takes, its interoperability within organizations, effects on dominant local languages, etcetera. Research has been particularly focussed within education and corporate interpersonal and communication activities, rather than within the $\mathrm{PR}$, marketing or even media sphere.

As I state throughout this thesis, I believe there are many areas of research that may be justified to improve or support communicability, understanding, audience reaction and other functions. It is necessary to adopt an interdisciplinary approach 
to consider an issue that would affect a specific discipline at its nexus that, in turn, would have a limitless impact by dint of more articulate, powerful messaging. This research is just a tentative stake in the ground. Space limitations preclude a detailed analysis of this perceived knowledge gap and the broader research projects that could be developed and implemented to hopefully contribute to the body of knowledge and support, or demolish, some of my perceptions and suppositions.

It would be wrong to state that a specific article is central to my research and underpins the whole necessity of ELF within PR activities. Certainly, there are many influential articles that act as important guides and no doubt further research will highlight many others, possibly from unexpected quarters.

Within the specific and focussed niche of the role of ELF within PR, I submit that more research is needed to consider the specific role language may play and how it can be better deployed, where necessary. The changing role of PR and its new positioning may also pose challenges that should also be considered, as this will impact also on language usage and deployment. Whilst the solution may sound simple - 'use better language' - I do not believe it to be a simple challenge, even though much positive change may be achieved through a simplified process or framework being developed and deployed. 


\section{RESEARCH FRAMEWORK}

To consider the role ELF may play in PR activities and establish elements of validity towards my hypothesis, several research elements are necessary. At this stage, it is only possible and practicable to test certain aspects within one country, and it is expected that findings may vary between country and various research groups located within it.

I do not believe there is a clear, sole theory that can underline my research, since it is not a case of 'using $x$ theory to analyse $y$ event'. Basic Linguistic Theory may be the closest relevant, since it describes a theoretical framework and concepts that have been used to describe languages and linguistic usage. Discussing linguistics theories', Dixon (1997) wrote 'The major development has been the invention of a number of restricted sets of formalisms, that have been called 'theories'. Each is based on some part of Basic Linguistic Theory. Each is useful for describing certain kinds of linguistic relationships, but it is put forward as if it were a complete theory of language. The word 'theory' is being used in a novel way.'

My research is business- and media-led in focus. I argue that it is not valid to focus on the linguistic structure of individual content (such as a press release) with the strict reliance of a stated theory: this could have relevancies if one was examining tone, framing and similar factors. However, the research does consider elements that may contribute to the theory.

It is important to reiterate that this research draws on linguistics but the intended result is intended for practical deployment within the business community, where comprehension is key, and this can be ultra-subjective in nature.

9 There are theories related to ELF (Guido and Seidlhofer, 2014), although their relevance and utility is less apposite, which views linguistics as a tool and focusses on elements such as phonology and pragmatics, rather than the core subject for examination. Deeper analysis of ELF from a linguistics perspective was purposefully left out of the literature review and the foregoing is only mentioned for purposes of completeness. 


\subsection{Research approach}

I have purposefully adopted a mixed approach to research, being part of an openended strategy that incorporates both deduction, presumption and induction. In other words, to provide an example, you may deduce that clearer language is desirable for effective communication, you may presume that a company would not knowingly impede this desire and induce through new research how language is being used and consider ways to improve matters.

The focus of the research is the organization and its operational behaviour, although it must be remembered that organizational behaviour can be influenced by social phenomena both internally, such as its personnel, and externally, for example being the society in which it operates.

In this research, I sought to deploy evidence-based management, using '...the best available evidence to improve management practice' (Reay et al., 2009). This evidence utilises four sources of information - practitioner expertise and judgement, evidence from the local context, critical (systemic) evaluation of the best available research evidence and perspectives of those who may be affected by a decision (Bryman and Bell, 2011, p. 8). The evidence-based approach has been criticised by some for possibly favouring certain types of research and restricting what may count as legitimate research (Learmonth, 2008), as well as its risk for bias and other limitations. In this case, I feel that such concern is not valid, as the research is directly aimed at gathering information from those who undertake the work under research, as well as considering their typical output to hopefully test a hypothesis and lead to additional research.

Throughout the process, a certain degree of flexibility has been necessary, partially due to the exploratory nature of the research but also to '...remain flexible and to modify and adapt [...] research plans in response to opportunities and problems that arise' (Bryman and Bell, 2011, p. 15). I chose to embed the research in wider contextual factors and sought not to practice it in a vacuum (lbid., p. 16), so whilst it can never claim to be wholly comprehensive or definitive in nature, a degree of relevance, utility and practicality can still exist. 
The approach taken was a mix of epistemological and ontological (Ibid., pp. 15-23; Collins, 2010, pp. 36-37), the latter posing a bit of a dichotomy since two aspects of ontology - objectivism and subjectivism - may be relevant and can be opposing forces at times. Collins gives an example of objectivism that design is objective and that designers perform similar roles and are subject to job specifications and procedures to which they must adhere, whilst subjectivism holds that social phenomena are created from perceptions and actions of social actors and are in a state of revision.

Mixing different process elements, 'thrown together in grab-bag style as if they were all comparable terms' (Crotty, 1998, ch. 1), should this exist, may be considered risky but arguably necessary, as a piece of the bigger picture must be considered and this can only be undertaken using different tools or approaches.

I argue that in PR activities both elements of ontology can be relevant and similarly epistemological matters cannot be ignored: they go together like a nuanced recipe that cannot be formulaic in nature. Specifically, I considered epistemological knowledge from the literature to assess what is believed to be known and accepted as valid, even though the specific research hypothesis and operational sector itself remains fertile territory for additional research. Ontological elements were evaluated, put brutally from the 'real world', as from this can further epistemological knowledge be derived. My quantitative research and limited qualitative research added to the latter, whereas consideration of the literature, particularly considering other disciplinary activity and knowledge, has a valid place at the same time.

\subsection{Research paradigm}

I believe that there cannot be a single research paradigm for this thesis and the broader direction of my intended research, although clearly elements from different disciplines and subjects may have their own specific paradigms or key influences. If anything, maybe this is a hybrid pragmatist-constructivist approach. 
The problem with this is that each paradigm can have a different ontology, epistemology and theoretical perspective, and this impacts upon methodologies and deployed methods. For example, the ontology (reality) is that every company wants to set itself in the best possible light (positivist) and how they achieve this may be interpreted differently (constructivist) since there is no single reality or truth and individuals shape this creation in groups. This may be impacted by reception from internal stakeholders, colleagues and latterly external publics too who can take a similar constructivist approach, and the reality is that there is no one-sizefits-all situation and that reality is constantly changing, being renegotiated and interpreted (pragmatist) and in some ways perception-based (subjectivist). Naturally these 'realities' may also be derived by, or impacting, socially constructed entities that are under constant influence (critical). Add to the mix that there are several stages within the operation and these can share different values or ontologies that in turn are shaped by different epistemologies and certainly various theoretical perspectives, methodologies and methods.

It may be no surprise that no paradigm has emerged as being pre-eminent or preparadigmatic, if you assert there are many competing paradigms but no supremely defined ultra-paradigm with a 'capital P'. I am not yet convinced that Burrell and Morgan's multiple paradigm model is even entirely sufficient (Bryman and Bell, 2011, p. 25), although it may be influential, and in any case this entire area is the subject of open debate (Ibid., pp. 629-634).

I want to look at the very heart of the matter and consider how the 'building blocks' are formed and deployed within PR messaging; the outcome can have a much broader relevance and impact. Some of the deeper theory about linguistics may have relevance to how individuals react to language, which is a benefit within PR activities, although this would be rather specialist and more in keeping with a linguistics-led piece of research. My research is more focussed on the apparent complexity and formation of deployed language, and whether this is a barrier to accessibility and other post-consumption actions, which hopefully leads to deeper and broader research later. Thus, I argue, I must leave no stone unturned in the quest for knowledge and deploy liberally all tools, theories and more at my disposal. 


\subsection{Discourse analysis}

There are subtle differences between discourse analysis and critical discourse analysis. It does not help that discourse analysis is understood differently across the social sciences, due to different analytical and theoretical approaches being deployed that can be significant and more visible in certain areas. No doubt my interpretation and deployment may stretch it in the minds of some, although I believe it is valid, even if I do liberally merge both concepts. An element of criticality remains, even though the criticism is more of an advisory, supervisory concept to encourage improvement, instead of being anything else.

Fairclough promotes a three-dimensional model for discourse analysis (Fairclough, 2001, pp. 20-30) that has influenced my research view, since he notes that discourse involves 'social conditions' that can be specified as 'social conditions of production' and 'social conditions of interpretation' and relate to three different 'levels' of social organization, namely 'the level of the social situation, or the immediate social environment in which the discourse occurs; the level of the social institution which constitutes a wider matrix for the discourse; and the level of the society as a whole' (Ibid., pp. 20-21). Fairclough (2010) also justifies critical social research 'which is focussed on better understanding of how and why contemporary capitalism prevents or limits, as well as in certain respects facilitating, human well-being and flourishing' and clearly anchors discourse analysis into this description. Business success is arguably a component of this.

Whereas discourse analysis can be considered as a generic term for approaches to analyse written (and other) usage or events, critical discourse analysis is explicitly anchored to being relational, dialectical and transdisciplinary (ibid.). Fairclough states that whilst it is a relational form of research that does not primarily focus on entities or individuals, for example, he does acknowledge that it considers social relations that are very complex and can also be layered, concerning 'relations between relations'. A 'discourse' can be an entity or object, he notes, but it is itself 'a 'complex set of relations including relations of communications between people who talk, write and in other ways communicate with each other, but also, for example describe relations between concrete 
communicate events (conversations, newspaper articles etc.) and more abstract and enduring complex discursive 'objects' (with their own complex relations) like languages, discourses and genres.' The relations themselves are dialectical and it is this character that makes it clear in Fairclough's mind that you cannot simply define 'discourse' as a separate object, even though it makes the concept of discrete, dialectical object relations a paradox on one hand, but when viewed as a power-discourse event it can become clearer ${ }^{10}$.

I believe that those who formulate PR messaging (and conduct other elements of PR/business activity) are individually affected by these three dimensions, as well as the company-at-large, and It can naturally impact on their creativity processes, their communication processes and their general demeanour and way of doing things, which itself is suitable for discourse analysis, whether 'critical' or 'Critical' in nature ${ }^{11}$. Fairclough called such interpretation a 'realist approach', and I am pleased to concur and anchor my support accordingly. It can be visible in their PR messaging too. A similar phenomenon can exist for individual media consumers of the PR messaging, as well as for any gatekeepers who may interpret it for their own publics. To expand my hypothesis, deeper analysis would be necessary, including a comparison to PR activity in another, differently structured country as well as considering different audience groups.

The emphasis of the role of language as being a power resource may be a nuanced, important difference, as it has the potential to affect the entire communications chain from origination to reception, with ELF perhaps weakening the use or specificity of linguistic terms that may be present in NS communication. This over-simplicity can, however, be a positive in many situations.

10 Fairclough's explanation is quite detailed, yet abstract in nature, and covers several pages. Reference to this text is highly recommended to get a deeper understanding.

${ }_{11}$ A slight piece of linguistic word play, to differentiate for purists between the formal term 'Critical Discourse Analysis' and the act of being critical to discourse under analysis. For my purposes, as stated, both terms should be interchangeable. 
Fairclough (2010), when describing what critical discourse analysis is, was clear to state that it is not analysis of discourse in itself - as many may assume - but the analysis of 'dialectical relations between discourse and other objects, elements or moments' as well as analysis 'of 'internal relations' of discourse', which is interdisciplinary or transdisciplinary in nature.

My argument for this choice of approach in my analysis is that it is not necessarily the words themselves, as important as they can be, selected for use within PR activities, but the nature and construction that may accompany them. I believe that this is more acute when ELF is added to the mix, whether as originator of the messaging, recipient or both, with it being possible to reformulate the messaging to improve clarity, readability, receptivity and future usage. My research draws in data that can be used to form part of this analysis.

Even though discourse analysis is often said to be more useful for qualitative research analysis, I believe that it has an impact on some quantitative elements too, at least when it is analysing the output of creative work (as in my thesis). Therefore, I argue it is beneficial to try and 'think' with a form of discourse analysis on both types of data, where available, as it will aid the overall research process. It is not a case of analysing the words deployed, such as are certain words inflammatory, defensive, reactive, etcetera. Instead the structure of the language is the focus, considering its broader scope to inhibit communications to some publics. It may even be an 'invisible issue' when the language appears to be of a technically high-standard.

Details of the specific approach and motivation for the research conducted in this thesis is detailed in a subsequent chapter. 


\section{RESEARCH METHODOLOGY}

To try and get a better understanding of how companies communicate, within the confines of this thesis, I conducted two distinct research projects to gather data.

It is acknowledged that there may be some concerns about the appropriateness of the research methodology deployed and its fit to stated research questions, but it must be underlined that this is an exploratory analysis to discover if there is support to my stated supposition and then whether it can be remedied through subsequent activity. Even within a clear, understood problem there would be limitations by examining a single country and a limited subset of participants, even if it may yield valid data. This issue is expanded upon in chapter 5.4.

\subsection{Common research pool}

With the presumption that the largest companies in Finland are more likely to export products or services, and have experience of international trade and nondomestic PR activities, I defined the research group as the top-100 companies, ranked by turnover ${ }^{12}$. Based on preliminary research, companies that clearly were primarily domestic ${ }^{13}$ in focus, such as retail companies and educational institutions, were excluded.

Between 50 and 75 per cent of the companies in the research group could have export-based activity of various levels, the matter complicated by foreign ownership, nature of trade and their focus of business presently. In some situations, the companies themselves may own subsidiaries that are more exportfocussed, yet their presence is consolidated within group financial reporting, as

12 Source: statutory data, compiled by Largestcompanies.com and accessed in March 2017.

${ }^{13}$ It can be argued that there may be a secondary level of export activity, such as the University of Helsinki selling a Finnish education to foreign students, but that starts to become a semantic argument over the place of supply and definition of export, befitting an economics thesis or similar. For this thesis and its research, such omissions are immaterial. 
evidenced by the research group's list. Many of these excluded companies can have, in any case, a need for English language communications, made using ELF.

It is acknowledged that what the larger companies may do is not necessarily reflected in the behaviour of all companies. Some smaller companies may do 'bigger and better things' within their narrow confines, whilst others may be effectively in ignorance of best, or accepted-practice. By selecting the larger companies as the sample, the justification is that they have the greater possible desire, financial resource, internal knowledge and potential to undertake what could be collectively viewed as best or accepted-practice, whilst being also open to emulating what their smaller brethren may undertake if necessary. I structured the research so that the same models could be built upon, if desired, in future research.

I do not believe there is a specific risk or bias in solely examining Finnish companies at this stage, particularly in research that is primarily exploratory in nature, and the disciplined focus of this thesis. According to the EF English Proficiency Index (EF Education First, 2016a), Finland is ranked fifth (out of 72) countries with a 'very high' proficiency of English and a separate fact sheet notes interesting generational and genus differences (EF Education First, 2016b). It may be inferred that Finnish companies should, therefore, have a good technical understanding of English that is reflected in their ELF-deployed PR activities, making it a suitable research cohort. However, as indicated within the literature review, this near-native understanding may work against it too, but this cannot be determined within this thesis.

Ethical issues were considered but none were particularly relevant. Companies are not identified as it added no specific value to highlight an individual press release and purported deficiencies or areas of possible improvement.

\subsection{Qualitative study}

A representative sample of 20 companies was taken (at random from the research group) and a senior communications executive invited to participate in a 
telephone-based interview. Despite reminders being sent, only two companies agreed to participate - most did not even acknowledge the inquiry. This created a limitation and changed how the study could be utilised. I accept that this may appear pessimistic, but it did reinforce certain observations that I have noticed in the past when researching Finnish companies and their international PR activities.

I have viewed it instead as a successful pilot test that yielded valuable directions for future research, rather than a specific failure, and it also justified the initial approach of a multi-modal research process.

The data acquired from the participants was valid and informative, and has been remediated into a narrative that is presented in appendix 1 instead of being analysed in the following chapter.

An ethnomethodological approach would help understand how order is created through 'talk and interaction' (Gubrium and Holstein, 1997). Three features are highlighted by this kind of research (Bryman and Bell, 2011, p. 393), namely an inductive view of the relationship between theory and research (the former is generated from the latter), an interpretivist, epistemological position (understanding the social world through an examination of the interpretation of that world by its participants), and a constructionist, ontological position (that implies that social properties are outcomes of interactions between individuals, instead of phenomena 'out there' that are separate from those involved in its construction).

The process was designed to use a structured questionnaire, so that respondents could respond with limited intervention from myself. I hoped that respondents would describe processes deployed, specific motivation for decisions taken and identify their viewpoint and valuation of various elements relevant to the research. As the original research intention could not be concluded, the questionnaire is not appended to this thesis. The findings, even in a reduced form, are valid and reflect credibility, which parallels internal validity, transferability, which parallels external validity, dependability, which parallels reliability, and confirmability, which parallels objectivity (Ibid., pp. 400-401). Justified questions can be raised about the validity and reliability of this research (Ibid., pp. 400-402). 


\subsection{Quantitative study}

A piece of quantitative research was designed and conducted to provide insight into how language is used and whether it may have a bearing on communications efficacy and reach.

It was necessary to develop my own research model, since I had not discovered an existing model that would suit my intended research objectives. Any existing models or methods of research, such as, but by no means restricted to Canagarajah (2006), Dewey (2007), Flores (2013), Jenkins (2009), Jenkins et al. (2011), Jenkins (2015), Millot (2017), Ravazzani (2016), and Waisbord (2016), seem to be highly focussed within linguistic theory and other disciplines, which whilst valid, would overcomplicate matters and be arguably less relevant.

My intention was, should the model yield results, that it would be a simple affair that could guide future research and be field-implementable within a (latter) framework. As a non-linguist, the barrier also existed that I had to also understand and appreciate the given contexts, meaning that it should be similarly easy (or easier) for latter inclusion within any framework.

A form of critical discourse analysis was chosen, based around the semiotic analysis of linguistic structure. I used computer-based textual analysis, considering identifiable metrics and structural parameters, and each document (press release) was examined for 17 different linguistic elements or structures ${ }^{14}$ that gave indicative information about its structure that could have a bearing on eventual reader comprehension and other actions. The actual methods used are less relevant, since there is a variety of so-called readability formula and tests that can be deployed, each with their advocates and critics, as I outlined earlier. Details of the analysed elements are contained in appendix 2.

\footnotetext{
${ }^{14}$ This selection was made after investigating available options to consider what I believed to be key and relevant elements that could, in turn, be easily evaluated and implemented if relevant. An intentional bias was made to elements that could be understood by a non-linguist or specialist.
} 
Whilst not explicitly considered, it should be noted that a clearer use of language will aid existing and future computer-based artificial intelligence searching and processing activities.

Care must be taken in discourse analysis, since 'writing is an art, not a science, and you can't judge its effects by mathematics or with machines', and that anybody 'could write pure nonsense that would score well on a readability test' (Hart, 2007). Much justifiable critique can be laid on statistical analysis of texts, especially concerning readability, yet in broad terms such analysis may yield certain structural errors, indicate possible reader comprehension issues or just flag up as suggestions for an 'even-better polish' to a text.

The intended place where a text will appear, its expected audience and even its contemplated usage can make a difference, such as this thesis: despite being written in a style that is less formal and hopefully more readable than many of its peers, it will still have a style that would be different to a mass-education book, blog post or newspaper article. Components of a sentence can make a difference to how it is comprehended and possibly acted upon, dependent on many factors individual to the reader. If the reader needs to re-read a sentence to clearly understand it, this may be a good sign that it needs modification (LaRocque, 2003). Clearing away 'the clutter', whilst focussing on necessary constructions and other necessary elements, is a critical balancing act to achieve (Brookshire and Brundage, 2016, pp. 151-152).

I first conducted a trial of my theory to assess whether the proposed research may be of sufficient utility. I selected six companies from the research group (formed for the qualitative research) at random and collected six of their latest press releases (excluding purely financial reporting-related releases as they would be particularly specific and jargon-laden) in English, for examination. By selecting six press releases from each company, this permitted averaging of individual results that could provide data value, whereas individual releases could vary by author, 
context or even collaboration between parties ${ }^{15}$. It is my belief that this method of selection minimises bias and the risk of irregular data being present.

The actual content is less important as its utility, attraction and benefit to the recipient is very subjective. There is, however, an argument for making the content as understandable and 'linguistically attractive' as possible, especially when both sender and receiver are using a non-native language, to increase the likelihood that the message is delivered and possibly positively reacted to in one way or another. I also selected this method of research since I am not a linguist and it struck me as an interesting area to consider, to review my 'preconceptions' formed over the years.

After successful validation, I expanded this to 15 companies and define my motivation and procedure below. In this situation, the sample size was not viewed adversely, representing 15 per cent of the research group ${ }^{16}$. Within its cohort and within the broader group of all (potential) businesses, I consider it to be a valid source of additional data. The purpose is to get an understanding about one part of the broader hypothesis and to see the range in which it fell, before possibly other research is required to take this element further. In any case, it could be combined with insights from the qualitative research and theory to see if 'actual reality' mirrors up with 'projected reality' and 'best practice'.

The same model can be expanded later, with future research, to make greater statistical analysis of gathered data, especially comparing between research groups, that may yield additional insight.

\subsection{Validity}

I believe that this self-constructed methodology served a useful purpose for this research and would be a good foundation for future research into the subject and the development of any implementation framework. Some of the elements may be

\footnotetext{
${ }^{15}$ Analysis itself that is either not relevant to, or outside the scope of, this thesis.

${ }^{16}$ As calculated before companies who were not considered due to their lack of direct export relevance were considered.
} 
more relevant to the English language and those that share elements of its linguistic structure, although many other elements can have broader linguistic appeal and relevance.

The entire methodology of my research may appear unbalanced when compared to my original intentions. From the beginning, I knew it would be risky to undertake qualitative research with Finnish companies for the reasons stated earlier, although it was never intended to be the central pillar of the research but a useful adjunct. It still has this utility, albeit without as much amplification as I had desired.

The quantitative research was always intended as the primary focus since, to use an English idiom, actions speak louder than words, and in this case the way words are deployed within messaging is more important than the words themselves. Language cannot always be articulated in pure numbers, although it may give valuable indication when considered in conjunction with individual text. Calculated metrics or values, based on document analysis, can be mere guides and even their methodologies and intended uses varies. Data considered should be instructive, viewed as a guideline or objective, but blind observance should not be given to calculated figures at the cost of information transmission or intelligibility.

That said, this does not invalidate research validity, but it acts as a practical caveat that you cannot say 'aim for $X$ in value $Y$ for good communication alone' and there are times when rules are meant to be broken, to use another English idiom.

It is not the intention of this thesis to provide criticism of an individual company and its messaging output. It must be remembered that certain situations will demand a different use of language that may lead to an over-representation in certain metrics. This is one reason why the companies are not specifically named, nor identifying highlights from their messaging provided. 


\section{RESEARCH ANALYSIS}

In this chapter I analyse ${ }^{17}$ the quantitative research, considering the entire data set (otherwise referred to as data) to understand the scale of findings from the cohort. Data from six press releases each taken from the 15 analysed companies is averaged and calculated, allowing the company to be considered both individually and collectively within the cohort, in accordance with the details given in chapter 5.3 and presented with the caveats noted in chapter 5.4. I felt that this would give a clearer impression of a company and how it communicates, instead of focussing on one specific release that could be a particularly bad (or good) example from the company that is far from its norm.

Selected examples ${ }^{18}$ of higher, outlier data is provided to show usage, and I have highlighted data that I consider might not have been optimal in such circumstances. Measurement units vary between analysis elements, and that $\mathrm{Y}$ axes of charts cannot be compared as data is presented to highlight differences.

The data presented for each analysed metric may appear sparse. This is deliberate, since there is little utility in over-analysing the data, as there is never going to be a 'magic number' for each metric that a company should strive to be under (or over) within its message construction.

Individual circumstances will vary, although the data individually and collectively can help streamline a message and it will also indicate areas that may require closer attention and remediation. It is similarly important to present all data analysed, since many elements separately but collectively may impact on a piece of messaging in different ways, all due to different writing styles and other factors.

${ }^{17}$ For brevity and readability, the percentage symbol (\%) is used. Rounding is to two decimal places, which may create occasional rounding discrepancies. The mean has been calculated from the sum of individual company means, and may have a very minimal, immaterial rounding difference (both are tabulated in appendix 3).

${ }_{18}$ Underlining represents an example of the feature under analysis, an ellipsis shows removed data and XXX indicates a redaction of a company name or other identifying mark. 
All have relevance in this thesis to help validate a hypothesis and would be forming part of any future, broader research to help identify possible differences in other countries, sectors and audiences.

There was nothing identified in the analysis that could explain a difference between company $A$ and company $B$, to justify a major difference in a specific research metric. The companies are relatively comparable in terms of resources and have similar potential. The creation of the research group removed any obvious differences, such as a company not having any real export activity, that could explain a more parsimonious attitude to PR messaging in English.

It was not possible to analyse specifically the data through the prism of Fairclough's three-dimensional model, despite it being influential, although I do maintain that the data under analysis has been produced by this model, and the influences that it would leave on the author(s). Similarly, the consumers/readers of the content will also have their own individual filter or validation using his model. Therefore, put bluntly, making the data as accessible as possible would be desirable, as all involved are humans and thus not 'set up' the same way. All the data elements are meaningful, even if results may appear to be similar, since it reflects the behaviour of a defined cohort, and other definitions can exist for similar cohorts too (but that is outside of the scope of this thesis).

\subsection{Statistical analysis}

The use of weak verbs varied considerably, representing $5.90 \% \mathrm{MIN}^{19}, 44.80 \%$ MAX $^{20}\left(\right.$ RANGE $\left.^{21}=38.90\right)$ that can be viewed as $Q_{1}{ }^{22} 17.60 \%$, mean ${ }^{23} 24.35 \%$, median ${ }^{24} 24.15 \%$, and $\mathrm{Q}_{3}{ }^{25} 30.00 \%$. The range is skewed by a small subset of data. Mean figures are shown in figure 2.

\footnotetext{
${ }^{19}$ The lowest value observed.

20 The highest value observed.

21 The spread of data observed.

22 First quartile, whereby $25 \%$ of the data is less than this value, see http://www.mathwords.com/f/first_quartile.htm/.

${ }^{23}$ The average observed.

24 The mid-range point of all data when sorted by value.
} 
Examining the higher occurrences of weak verbs, it was noticeable that the underlying releases were written quite passively. Pre-packaged speech quotations appeared to be full of weak verbs that significantly contributed adversely, e.g. 'It is important to find ...' and 'XXX have may be far away, and it is important that ... present when they are needed'. It is an area that should be more closely monitored as it did inhibit the flow of the message in many cases and created a barrier to continue reading.

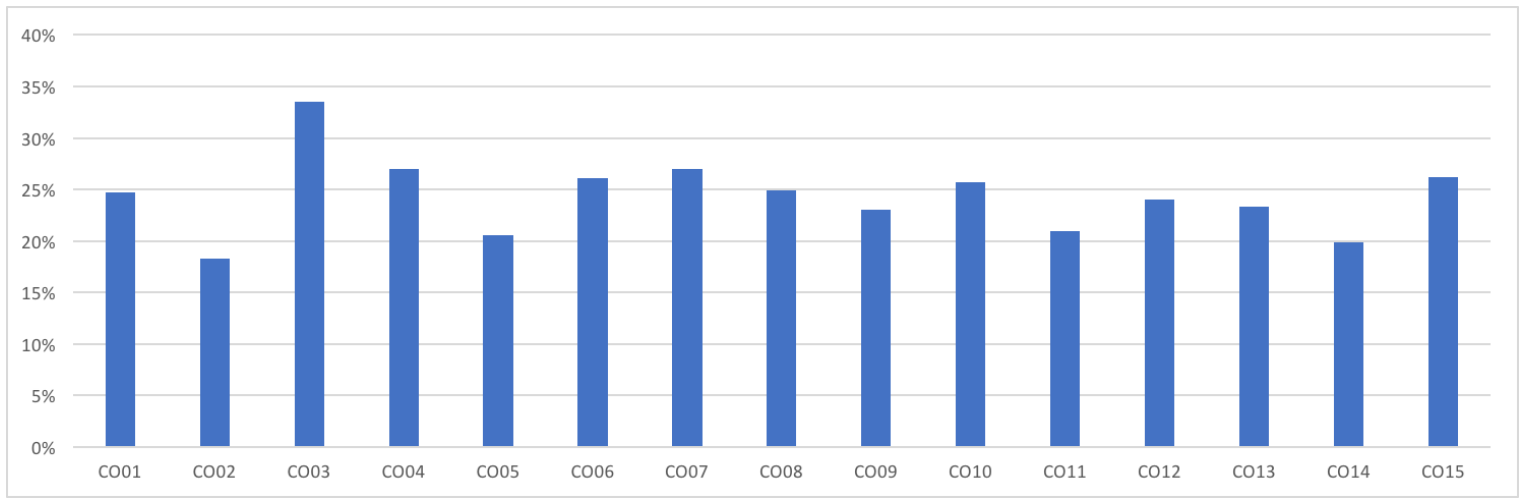

FIGURE 2. Mean presence of weak verbs per company

Filler word usage was mostly comparable, representing $0.00 \%$ MIN, $3.00 \%$ MAX (RANGE $=3.00$ ) that can be viewed as $Q_{1} 0.30 \%$, mean $0.80 \%$, median $0.70 \%$, and $Q_{3} 1.20 \%$. The range is skewed by a small subset of data. Mean figures are shown in figure 3.

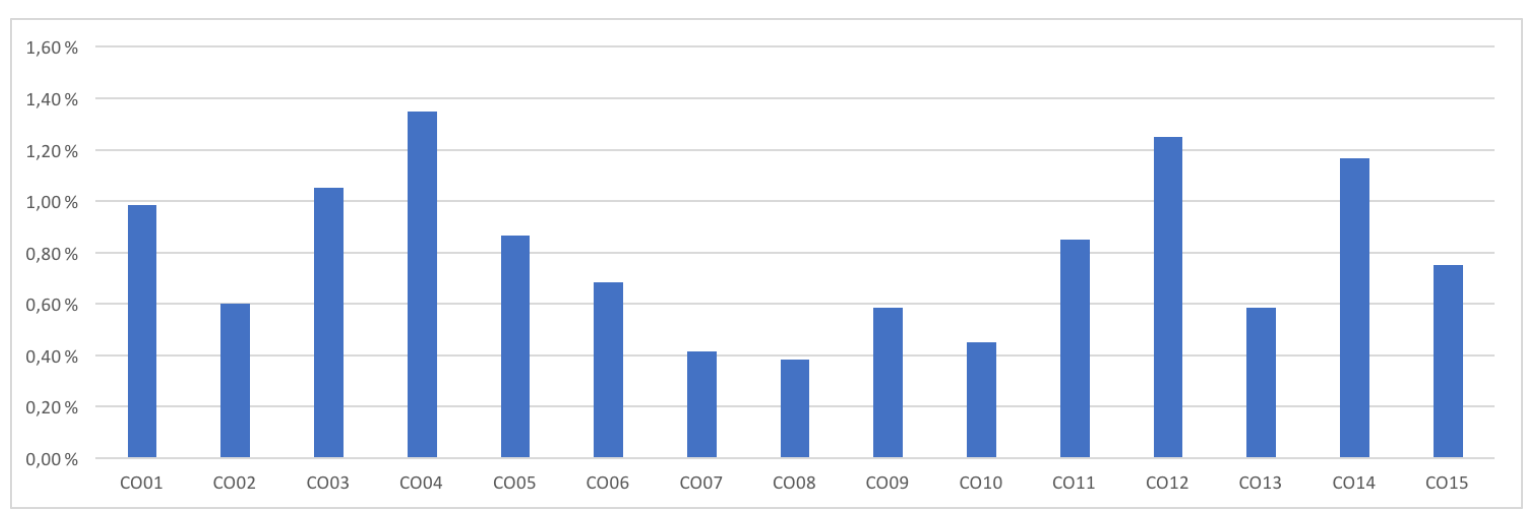

FIGURE 3. Mean presence of filler words per company

25 Third quartile, whereby $75 \%$ of the data is less than this number, see http://www.mathwords.com/t/third_quartile.htm/. 
The worst 'offender' had broken away from a traditional press release format, using instead a more conversational tone, posing 'questions' to preface the answers (core information they sought to disseminate). In other situations, filler words were primarily extraneous, innocent in themselves such as 'completely eliminates', 'much lower downtime' and '... is scheduled to start during ...'.

Occasionally it was a less-optimal linguistic construction, such as '... how to get started without disturbing operations heavily'. Considering the typical size of a press release and the relatively low percentage figures, appearance of filler words may not be of primary concern.

Nominalizations were broadly clustering in groups, representing $0.00 \% \mathrm{MIN}$, $26.50 \%$ MAX (RANGE=26.50) that can be viewed as $Q_{1} 8.30 \%$, mean $12.13 \%$, median $12.55 \%$, and $Q_{3} 15.60 \%$. Mean figures are shown in figure 4 .

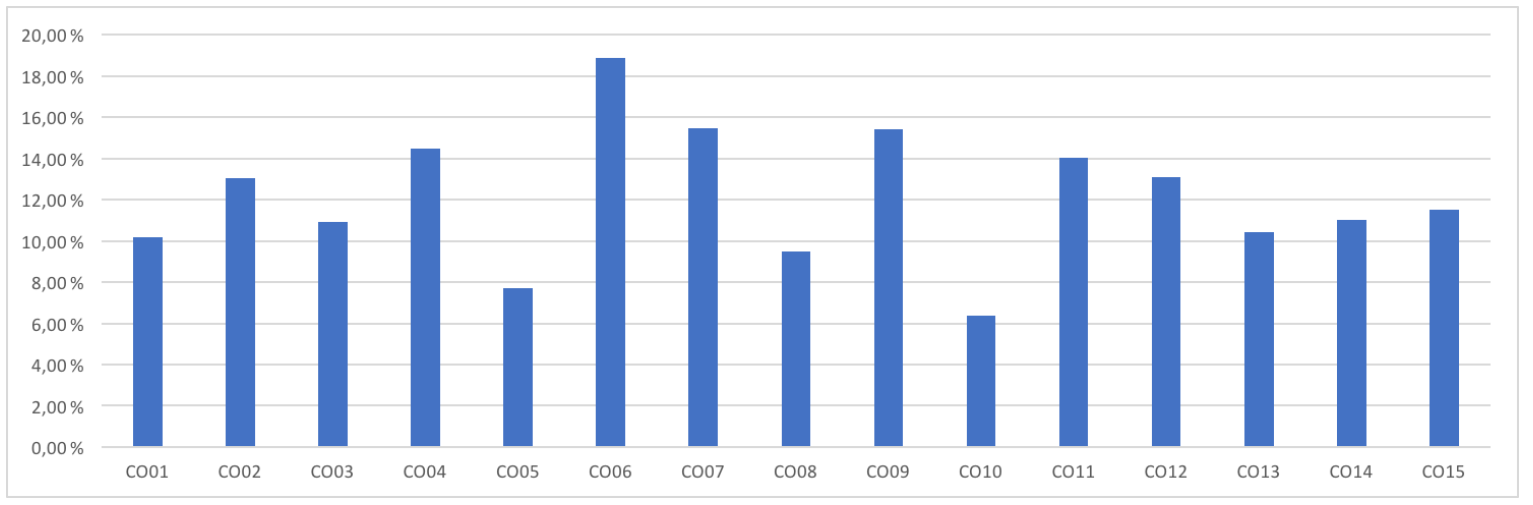

FIGURE 4. Mean presence nominalizations per company

In many cases usage of nominalizations appeared justified and relevant to the type of message being disseminated.

Caution is advised to help 'de-jargon' text where possible, and this may be aided by the deployment of a clearer writing style. In some situations, it can be imagined that the text was translated into English, creating an additional layer of difficulty.

One example of a particularly nominalization-laden text that could also have benefitted from redrafting is '... the same time, the challenging economic 
conditions and changes in the business environment are affecting the company's traditional businesses to the extent that the company has had to hold statutory employer-employee negotiations with regard to recognising operations and reducing the cost structure.'

Entity substitutions were similarly broadly clustered together, with some exceptions, representing $0.00 \%$ MIN, 14.30\% MAX (RANGE=14.30) that can be viewed as $Q_{1} 1.60 \%$, mean $4.07 \%$, median $3.05 \%$, and $Q_{3} 5.80 \%$. Mean figures are shown in figure 5.

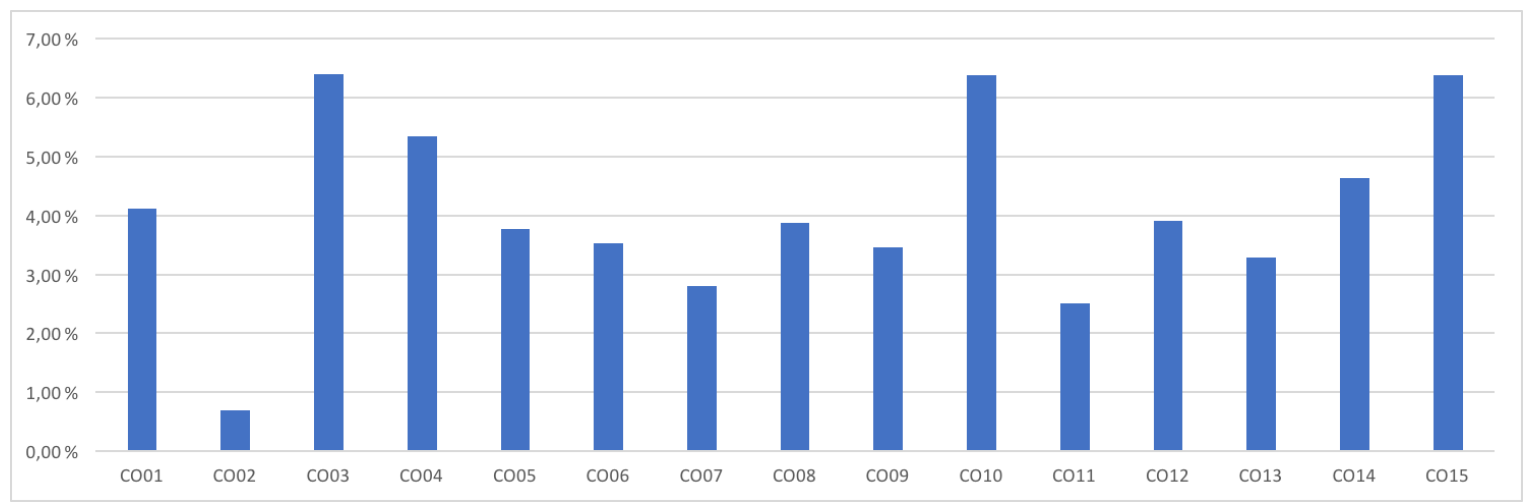

FIGURE 5. Mean entity substitutions per company

In many situations, these can be hard to avoid and feature particularly in quotations where 'we are very pleased' and 'we are positive that'-type terms feature. Some entity substitutions can be difficult to remove and/or have their place, e.g. 'encouraging that so many...' and 'continued confidence among them and our clients'. In conversational-style text it can be avoided if words such as it, there, you and we are restricted.

Negations were broadly less troubling, representing $0.00 \mathrm{MIN}, 0.50 \mathrm{MAX}$ (RANGE $=0.50$ ) that can be viewed as $Q_{1} 0.00$, mean 0.05 , median 0.00 , and $Q_{3}$ 0.10. Mean figures are shown in figure 6. Mostly their use appeared to be justified, such as '... have not yet registered...' and '....related to the so-called unclaimed shares.' 


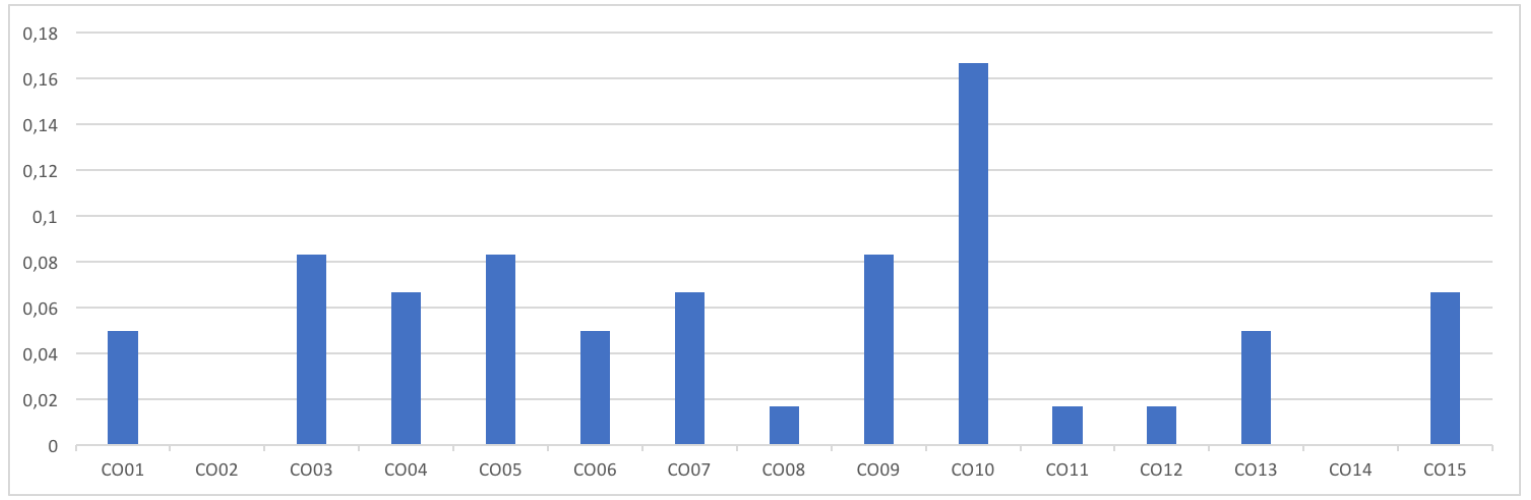

FIGURE 6. Mean negations per company

Noun clusters potentially posed issues for some companies, representing $6.10 \%$ MIN, 46.90\% MAX (RANGE=40.80) that can be viewed as $Q_{1} 18.250 \%$, mean $26.16 \%$, median $26.70 \%$, and $\mathrm{Q}_{3} 34.33 \%$. Mean figures are shown in figure 7 .

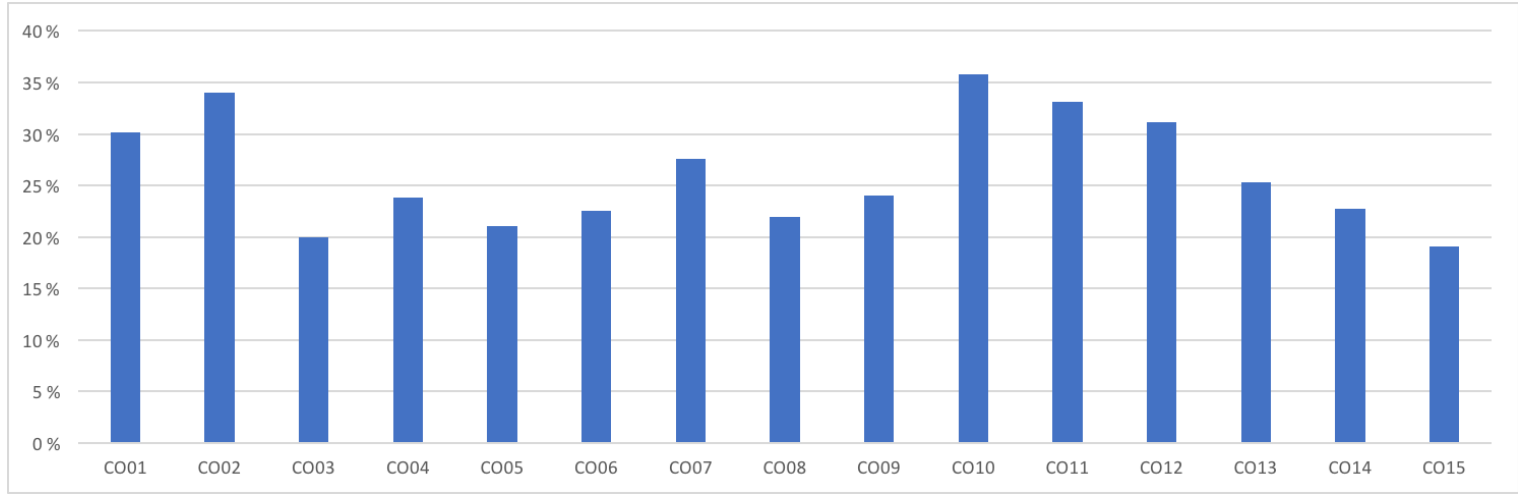

FIGURE 7. Mean noun clusters per company

Some usage could be described as justified but jargonised or specialist terminology, such as 'passenger load factor', 'unit revenue growth' and 'example of XXX's commitment'. Minor adjustments may be possible, as it can otherwise inhibit readability in sentences such as ' $X X X$ announced plans to utilize all of its $X X X$ resources align strongly with $X X X$ 's strategy of $X X X-X X X \ldots$.

Passive voice usage was varied, representing $0.00 \mathrm{MIN}, 1.00 \mathrm{MAX}$ (RANGE $=1.00$ ) that can be viewed as $Q_{1} 0.20$, mean 0.29 , median 0.30 , and $Q_{3}$ 0.40 . Mean figures are shown in figure 8. 


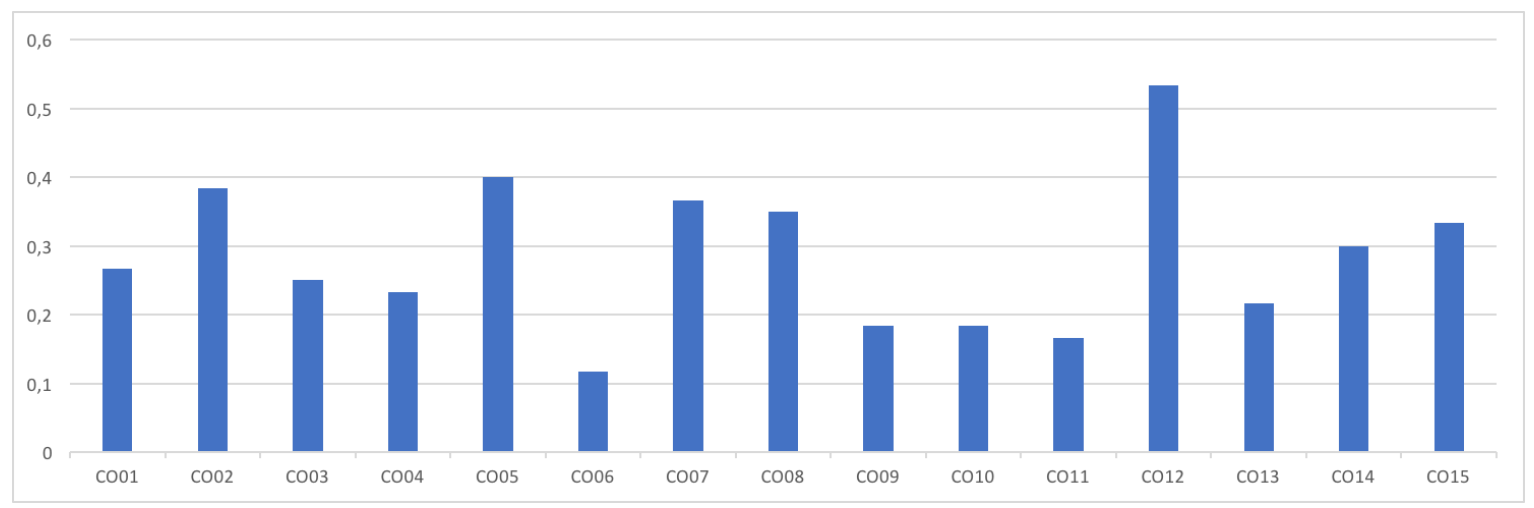

FIGURE 8. Passive voice usage per company

Passive voice can be clear and it may have its place, but it is worth reducing where possible through rewriting phrases, removing perhaps unnecessary accompanying adjectives. Sometimes repetition is also a problem, such as a contract announcement with a later paragraph mentioning 'XXX's announced plans' when 'XXX plans' would have sufficed.

Presence of modals did not appear to be significantly troubling, representing $0.00 \%$ MIN, 4.50\% MAX (RANGE=4.50) that can be viewed as $Q_{1} 0.70 \%$, mean $1.48 \%$, median $1.30 \%$, and $Q_{3} 2.03 \%$. Mean figures are shown in figure 9.

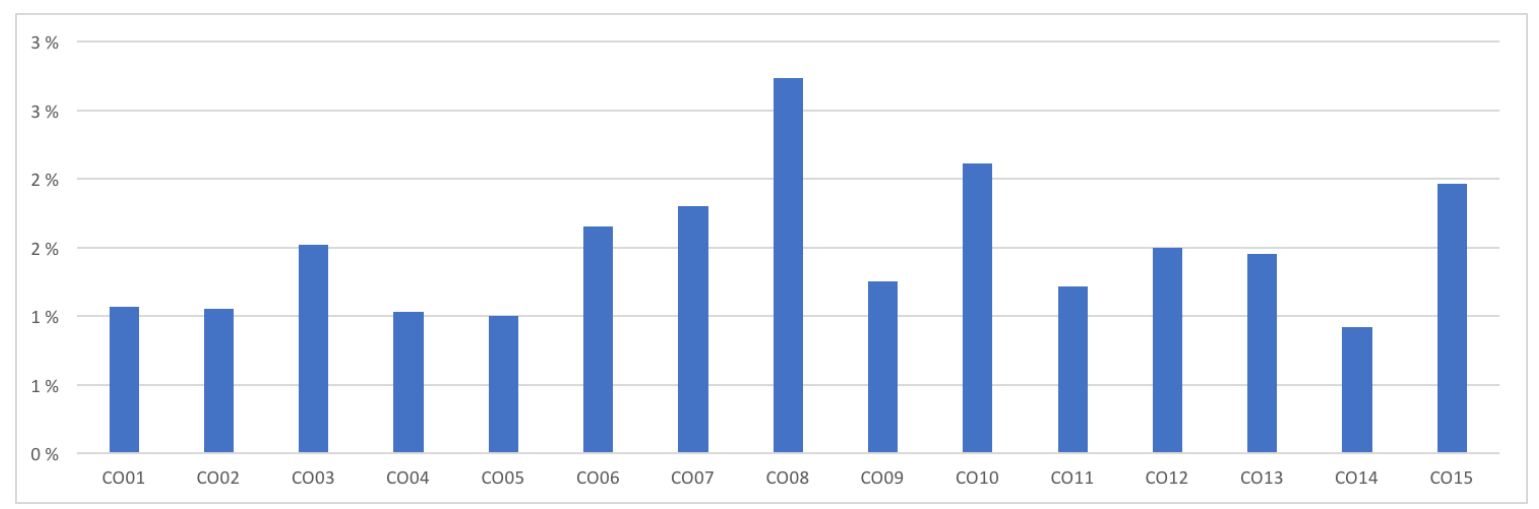

FIGURE 9. Mean modals per company

Most usage could be justified, for example a contract 'will bring', 'will allow' and 'will provide'. Some rewriting may reduce the number of modals and make the text appear less formulaic, especially when sentences are short, e.g. 'It will be a new warehouse. It will be ready soon. The building will store'. 
Rare words pose a few problems for analysis on one hand, as this can be contentdependent, and it is not unexpected that some releases may have higher values due to specialist terms, company names and so-forth, although if these are excepted it is good to have as low a 'rare word' score as possible.

As it Is, analysing the text in its entirety, this represented $6.40 \% \mathrm{MIN}, 33.70 \% \mathrm{MAX}$ (RANGE $=27.30$ ) that can be viewed as $Q_{1} 14.50 \%$, mean $18.98 \%$, median $19.05 \%$, and $Q_{3} 23.45 \%$. Mean figures are shown in figure 10 .

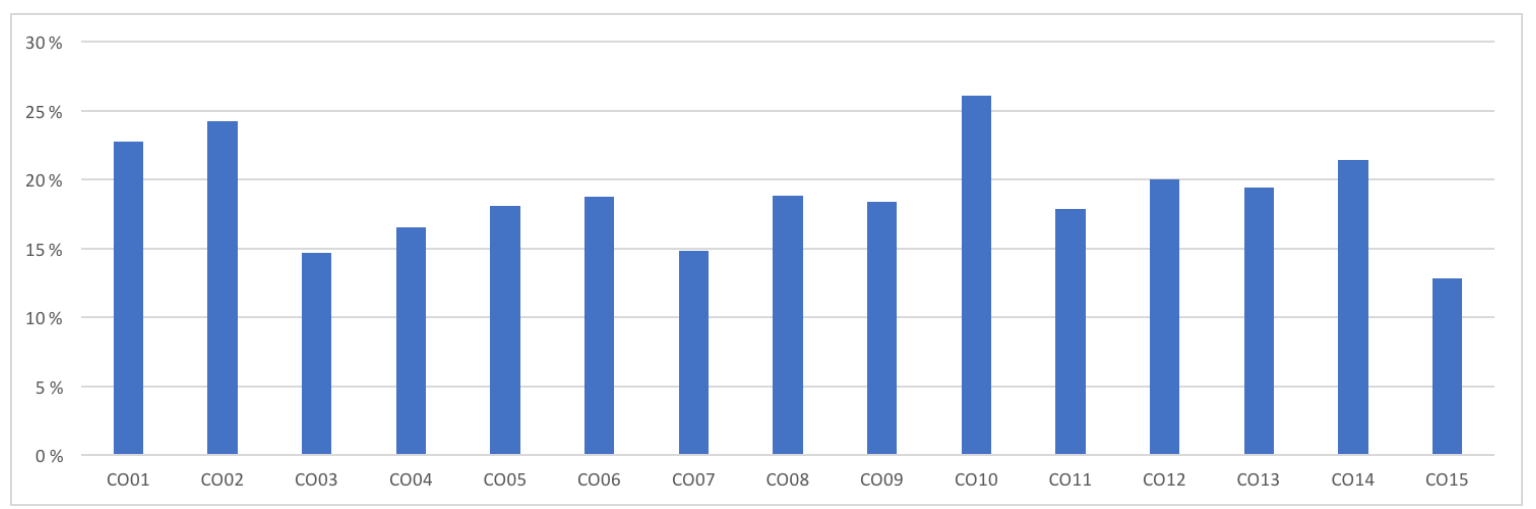

FIGURE 10. Mean rare words per company

A press release announcing a new appointment recorded the highest level of rare words, in part due to frequent repetition of the appointee's name, but also due to the presence of company names. No adverse reaction should be taken if a high rare words score is recorded, but a check to see if any words can be simplified where context and comprehension are not at threat, such as changing 'alleviate' to 'ease' can be worthwhile.

Extra-long sentences represented 0.00\% MIN, 30.0\% MAX (RANGE=30.00) that can be viewed as $Q_{1} 0.00 \%$, mean $3.84 \%$, median $0.00 \%$, and $Q_{3} 6.95 \%$. Similarly, extra-short sentences represented $0.00 \% \quad \mathrm{MIN}, 37.9 \% \mathrm{MAX}$ (RANGE $=37.90$ ) that can be viewed as $Q_{1} 0.00 \%$, mean $3.13 \%$, median $0.00 \%$, and $Q_{3} 3.58 \%$. Mean figures are shown in figure 11 . 


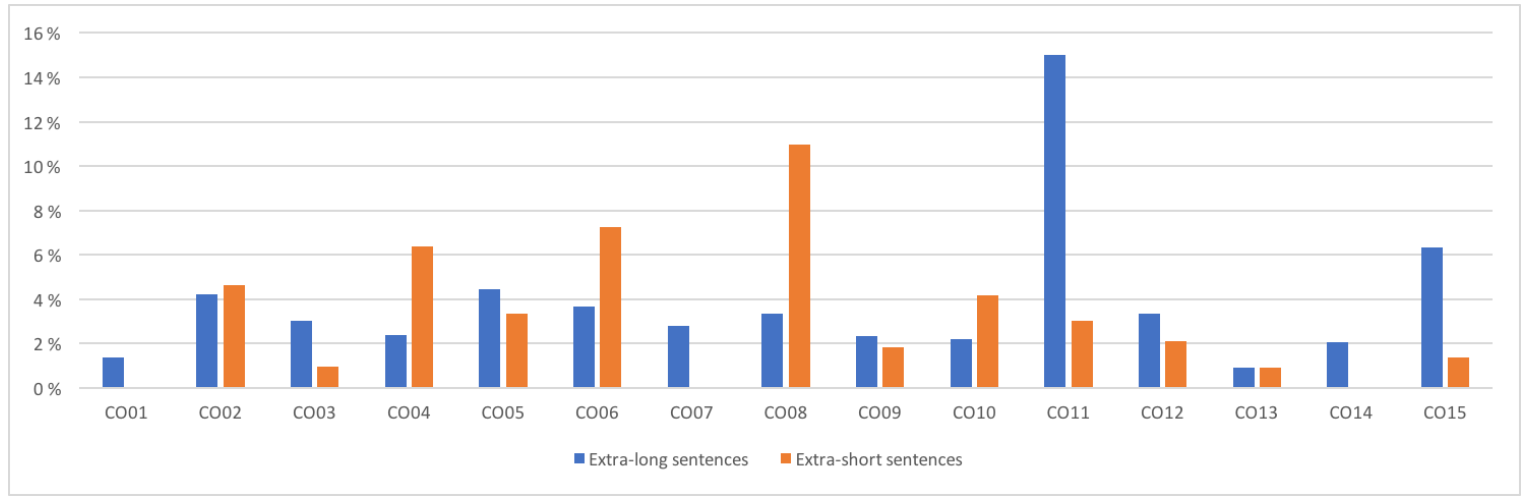

FIGURE 11. Mean extra-long and extra-short sentences per company

These can be problematic in certain situations, where there are plenty of clauses. Then use many shorter sentences. Context may be the key factor.

If the information communicated justifies the underlying text, no adverse reaction should automatically be made about the length of press releases, even though some guidance may be made for issue such as search engine optimisation and distribution over wire services. The structure may be more apposite.

Within the analysis, it is interesting to note that companies generally are relatively banded together, representing 49.00 MIN, 798.00 MAX (RANGE=749.00) that can be viewed as $Q_{1} 191.0$, mean 289.44 , median 281.00 , and $Q_{3}$ 368.0. Mean figures are shown in figure 12 .

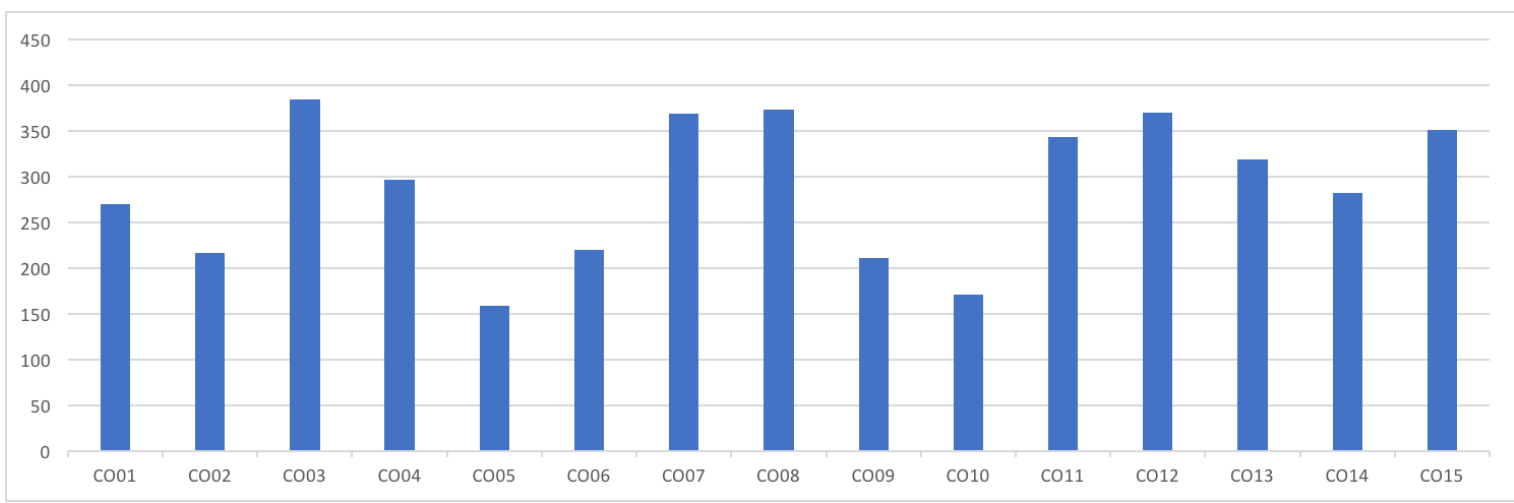

FIGURE 12. Mean word count per company

Words per sentence can have an impact, although there is not always a lot of wriggle-room. In any case, the analysed companies are relatively banded together, 
with a few individual releases skewing results at both limits, represented as 4.70 MIN, 34.40 MAX (RANGE=34.40) that can be viewed as $Q_{1} 18.68$, mean 21.17, median 21.17, and $Q_{3}$ 23.93. Mean figures are shown in figure 13. No additional analysis was necessary.

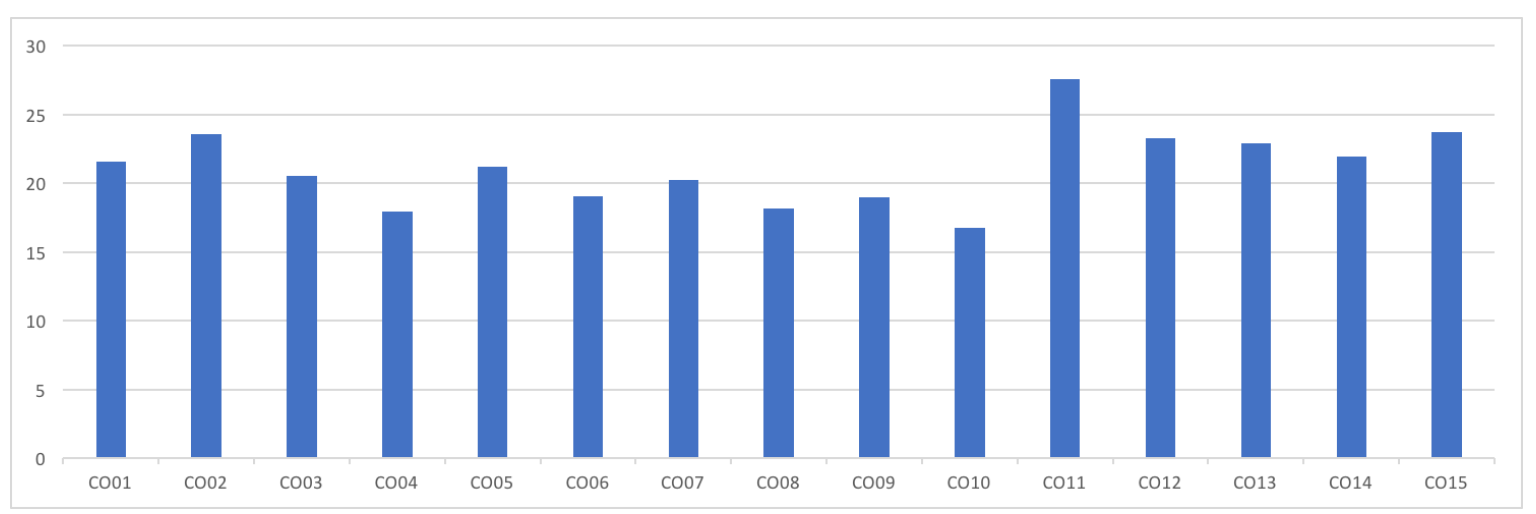

FIGURE 13. Mean words per sentence per company

Concerning readability, two specific formula were individually considered and a separate average of several additional formula made. Some individual releases skewed the values at the lower and upper ends of the ranges, but when compared on a company level there was a closer degree of banding.

Examining the Flesch-Kincaid GL readability, this was represented by $5.90 \mathrm{MIN}$, 19.80 MAX (RANGE=13.90) that can be viewed as $Q_{1} 12.08$, mean 13.29, median 13.29, and $Q_{3}$ 14.80. New Dale-Chall readability was represented by $0.90 \mathrm{MIN}$, 9.60 MAX (RANGE=8.70) that can be viewed as $Q_{1} 6.80$, mean 7.40, median 7.40, and $\mathrm{Q}_{3}$ 8.20. The Readability average was represented by $7.70 \mathrm{MIN}, 19.80$ MAX (RANGE=12.10) that can be viewed as $Q_{1} 12.90$, mean 14.06, median 14.00, and $Q_{3}$ 15.45. Mean figures are shown in figure 14.

As these are combinations of various analytical factors, further analysis was not relevant, other than to note the ranges deployed after ignoring an exceptional lower figure due to it being a very simple announcement of a single fact. 


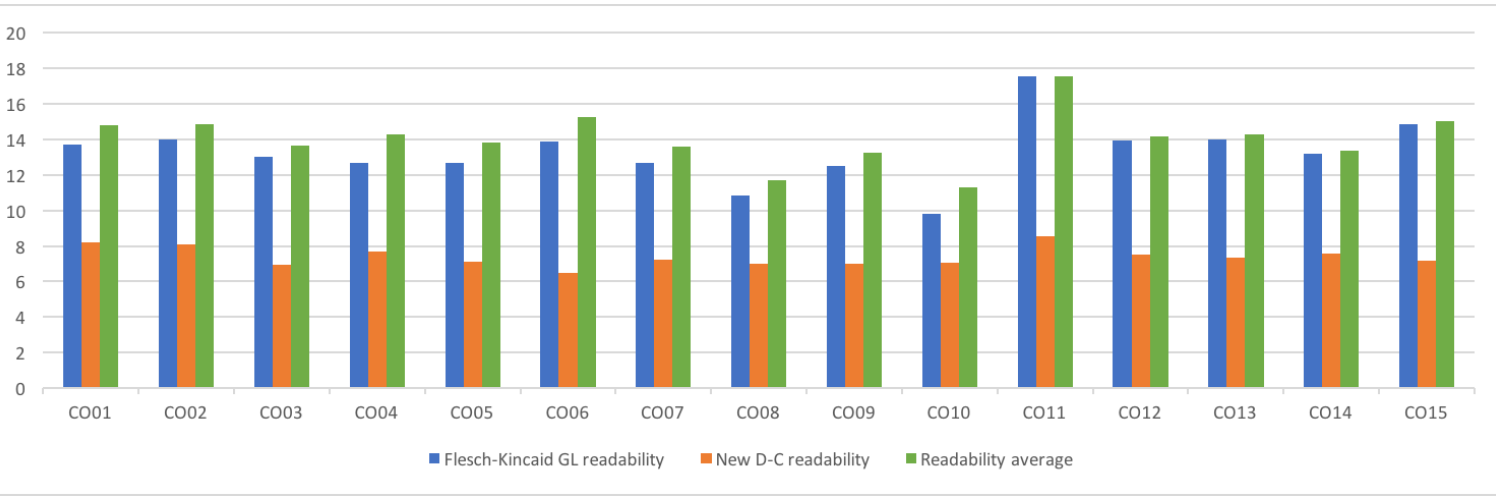

FIGURE 14. Mean readability analyses per company

Sentiment analysis is included as a piece of experimental data and consideration given to its possible validity. At best, present, it may be viewed being something worthy of consideration as a final check for an individual release. It is shown in figure 15.

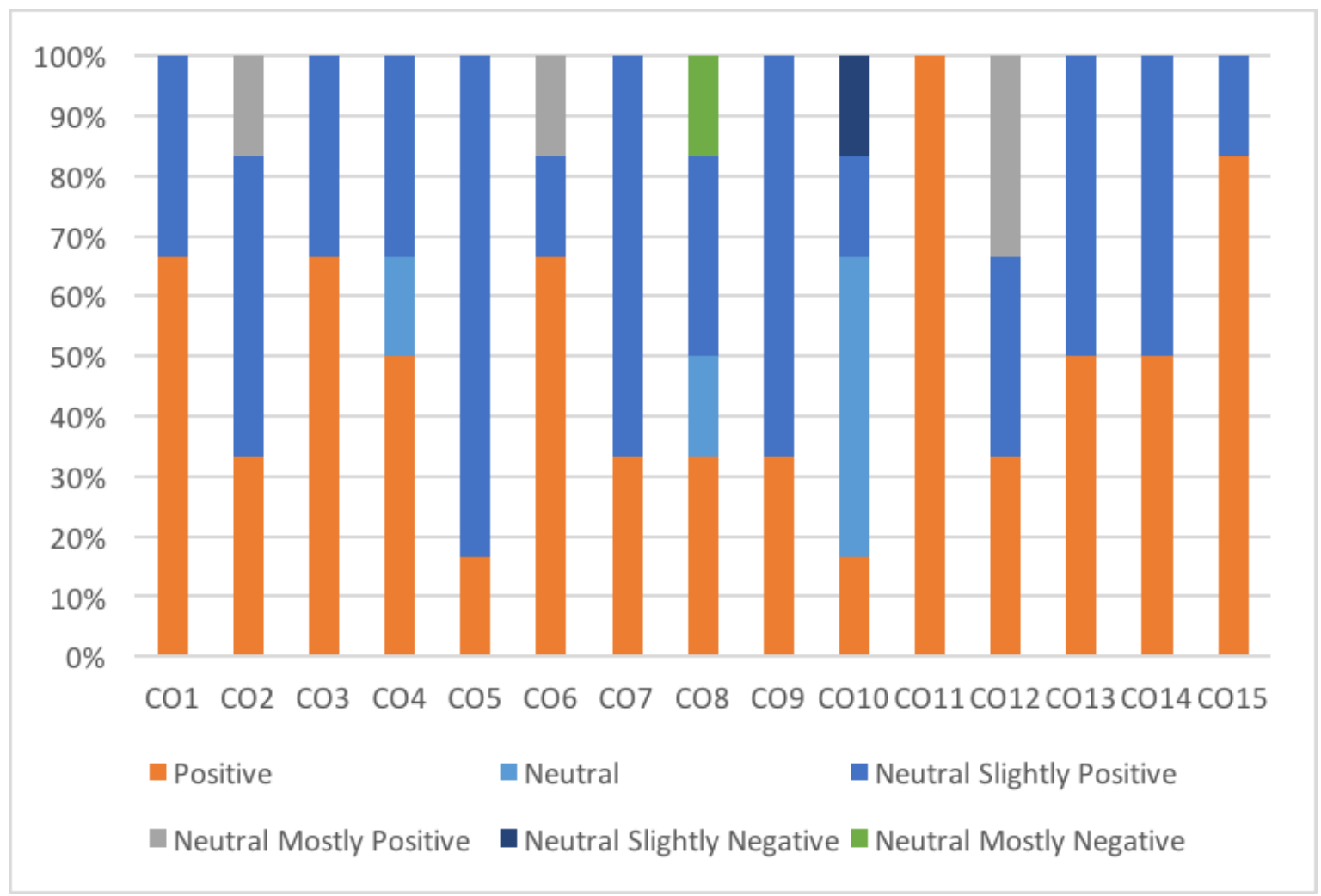

FIGURE 15. Experimental sentiment analysis per company

It was interesting comparing the data, however, over the cohort as one company recorded a 50 per cent negative sentiment overall, despite the individual releases 
not containing negative news, so some language modification and style amendment could be beneficial.

In the following chapter I discuss in context analysis of the research. As I have stated, there is no specific 'magic number' or key metric to discuss and react to. Similarly, as this is exploratory research, it is quite possible that other as-yet unconsidered elements may be relevant. However, I believe that this process has given a certain validity to my supposition and tentatively split the analysis and its data points into three positive action categories (that I have not yet formalised), namely 'should consider', 'could consider' and 'background consider'. 


\section{DISCUSSION AND CONCLUSION}

I do not want this to be a 'typical' thesis. My intention and hope is that it may be read by many, particularly outside of traditional academic circles. Notwithstanding hopes and realities, the aim of this chapter is to highlight an issue I believe to be important and to sow the seeds for possible change. It is not a closed subject and I believe much more research can be justified.

I would expect most communications professionals to have skipped chunks of this thesis, but if nothing else the first two chapters and this one should be essential reading and in turn it may inspire one to dig back into the details, such as analysis in the preceding chapter and the detailed literature review.

Considering the data derived from my research was on one hand challenging, whilst on the other hand it was indicatively interesting. Broadly one may summarise that the surveyed Finnish companies were relatively close to each other in terms of the linguistic content and quality of their press releases. It also supports the stated interdependent factors that influence ELF (figure 1, shown in chapter 3). This may not be a surprise, when you consider that the top companies are most likely to have the most experience in conducting PR activities and can dedicate resources to match.

Since ELF is an 'enabler of communications' (Louhiala-Salminen and Kankaanranta, 2012) and a 'language always in translation' (Pennycook, 2008), it is important that it is utilised effectively within all communications spheres, whether internal communications, PR or other activities where media is consumed (Blythe, 2006, p. 145; Kotler et al., 2009, p. 744; Kotler and Armstrong, 2010, p. 472). Obviously, ELF and communications-focussed activities can also play an important role within a company's internationalisation activities, both internally and externally.

Whilst not part of the stated methodology, I contrasted the findings-at-large to a press release announcing major and significant news from a regional, medium- 
sized company that has extensive export operations. It appeared to be selfproduced and I speculate that the author was not experienced with the production of such material, judging by its style, linguistic quality and overall readability. This would be supported by the observations of activities by Christiansen (2016) and shows the performance obligations raised by McWhorter (2007), Hallahan (1999) and Catenaccio (2008) to be lacking. Unless the recipient/reader is particularly interested in the content, after perusing the subject, I wonder whether a NNS would necessarily fight their way through the text and question how much some may comprehend. Further research into this area could be an additional fruitful line of inquiry.

In such theses, it is customary to restate in a discussion and conclusion relevant key theories and elements from the literature review. In a thesis that seeks to explore a new thought, which does not really have a lot of directly relevant theory or ultra-focussed literature at its core, this may be a challenge. It does not seem apposite to merely parrot relevant tangential theories and literature to support a hypothesis. In a conventional thesis, for example, examining the depiction of a certain group of people in a category of media, it can be much easier.

It is obvious to state that a well-written press release containing salient facts will be easier to comprehend, even if individual linguistics are less desirable, than a poorly written one. That said, the reader may not decide to spend time trying to decipher the indecipherable unless there is an acute need, so having a clear-tounderstand, accessible press release (or any content) should be obvious. In this situation, the combination of a self-check framework would have been helpful and, in conjunction with an improved linguistic quality, could have transformed this press release into a more readable, accessible and comprehendible entity.

As stated previously, broader research would be necessary to fully test my hypothesis and help identify the scale and scope of the perceived problem. It is quite possible that a similar analysis in a different country may reveal a different type of 'problems' with the construction of press releases. Similarly, there can be differences between the size of companies or even the sectors they operate in. This is before considering the average comprehension skills of target audiences in 
different countries. I do not believe that this will invalidate my hypothesis and recommendations, but it may lead to a more nuanced, complete framework being developed with various options dependent on extant circumstances.

Regarding the analysed data, it is important to reiterate that it is not a case of striving to reduce the value of individual metrics. Context must be the overriding factor. There are times when there will be a higher-than-average individual metric. It can be a cliché to say 'rules are meant to be broken', but still it can be valid. It may be better expressed through a good English quotation, of uncertain provenance, which sums up the situation well, saying 'Rules are for the guidance of wise men and the obedience of fools'. A thesis, for example, is a different body of text, and whilst you (should) strive for clarity and readability, certain linguistic structures may be necessary at times that may score adversely on the same metrics used to analyse a press release or other communication aimed for mass distribution to a global audience using ELF.

Evaluating and considering the individual metrics is one thing, seeking to sensitively reduce values is a wise thing, but there can never be a target number without making an argument to absurdity, sometimes referred to by the Latin phrase reductio ad absurdum that has fallen out of contemporary usage. Thus, the analysis of conducted research cannot explicitly interpret the data quantitatively to warrant specific conclusions, but it can give an almost qualitative-like sense to the quantitative analysis: this is not a misnomer.

It is important to state that the similar problems exist in other languages, as there is a perceived greater comfort in writing in one's mother tongue, so the 'issues' may be less obvious. In any case, it may 'sound right' and 'read right', but it is only when you seek to interpret it, perhaps through the lens of someone unfamiliar with the subject, less familiar with the language or even without the same level of enthusiasm or interest in the subject as the author, that issues become noticeable.

Each metric can have its own part to play within the analysis of a text, although I will here focus on a few key metrics that have the greatest potential to make change. Where the (original) language is of a high quality, this will be even more 
beneficial, creating a nuanced message that has the potential to have greater impact, comprehension and subsequent action through clarity and readability, as indicated by researchers such as Franceschi (2017), Hallahan (1999) and Louhiala-Salminen and Kankaanranta (2012). Naturally, simplification and clarity should be obligatory, noted McWhorter (2007).

The tone of a press release is important. I am a traditionalist, believing that it should be written in a similar style to a news story, using the 'five W's' approach ${ }^{26}$ - Who, What, When, Where, Why -- or a variant thereof. Some companies tend to use a more conversational style, but this feels to often stray into more marketingled 'brochure type' text that feels inappropriate and certainly less communicative. I argue that the press release can be repurposed to other uses but it can be harder to repurpose other text into a press release, without serious linguistic and copy surgery being deployed.

The presence of weak verbs (figure 2) was quite noticeable and easily remedied by changing from passive (indirect) to active (direct), which may give a boost or immediacy to the announcement where justified. Speech quotations can require greater attention. It can be a hard habit to break, but something that is easily flagged by an automatic check of text and then remedial action can be taken if necessary.

Filler words just get in the way. They often stray into the area of jargon, tautology or hyperbole. Figure 3 shows that some companies use words as 'comfort' for their text, in other words letting them support underlying text unnecessarily. A simple test can be to ask whether they add anything of intrinsic value. There is nothing wrong with precision in text, so why not say 'reduced downtime' instead of 'much lower downtime', or at least quantify it so there is meaning. The reader may have a different idea of 'much lower' than the writer, yet 'up to 75 per cent less downtime' can be transformative. Jargon also affected the presence of nominalizations, but a cleaner writing style generally will positively affect many metrics at the same time and need not obscure the core message.

${ }^{26}$ See https://en.wikipedia.org/wiki/Five_Ws/. 
Noun clusters (figure 7) potentially posed issues for some companies, where usage was not justified by specialist and necessary terminology. An assessment could be made about word choice and whether a better alternative may be available. The same can be said about so-called rare words (figure 10). It is inevitable that there can be an above-average presence of these, especially with those companies that may have non-English company, product and personnel names. The same issue will occur with text dealing with specialist terms that are common within the industry sector, but uncommon in the language corpus-atlarge. It is good to look at the rare words that are deployed, just in case anything can be simplified, where context and comprehension are not at threat, such as changing 'alleviate' to 'ease' can be worthwhile.

The length of sentences should be viewed as a guide (figures $11 \& 13$ ). Overall, the analysed companies had this reasonably under control. Quotations can be much longer. Some sentences need not be so long. It depends. See!

The typical length of press releases (figure 12) seemed to be suitable. If a company is going to use the release as a mould or basis for other communications, there is nothing wrong in making a longer version to gather information and then ruthlessly edit a shorter version for distribution as a press release. The analysis ignored the typical background information ('notes to editors' in typical press release jargon). Of course, this section should seek to follow the advice given concerning the main press release, but there is the potential to go into deeper detail about the company and its positioning, for example. It is a clear division in the release, just as an appendix in this thesis - it may not be consulted but it is available for those who are desirous of examining additional information at the same time.

The various readability levels calculated (figure 14) should only be viewed as guidelines. Here a lower value is, of course, better, since it suggests a broader pool of readers can better-comprehend its contents. The intended audience is key. A press release intended for the financial sector will be harder to comprehend than an announcement concerning a new sales director or the opening of a new store. Sometimes several versions may be better than one. 
It is not possible to give a specific recommendation concerning sentiment analysis (figure 15), although I expect that this is an area that will be more important in the future as underlying technologies are improved. It was an interesting experiment and certainly worthy of brief consideration if any analysis platform used supports it. If you believe you have a positive-sounding text concerning a major order and the analysis suggests it is neutral in tone, it might be worth just taking a harder look at it, particularly if you agree with other sentiment analyses that you have undertaken in the past. This can be very useful for NNSes who perhaps have a reduced vocabulary, but in reality, everybody can benefit from examining how others may view/react to a message.

What are the key things to consider? For this cohort weak verbs, noun clusters and the overall readability level were apparently most relevant, yet the other elements such as filler words, rare words and sentence length can be worthy of note. As you cannot know what the writer will write, all metrics have a relevance until analysed. Neither can you know how the reader will react with certainty.

My view mirrors that of Louhiala-Salminen and Kankaanranta (2012, p. 263), namely that language is not a factor to be forgotten and that it is an essential factor in the globalization of social systems, so one must get a better understanding of how PR works in society, of which business is a part of. Comprehended language can also boost cultural knowledge, enhancing trust and rapport building, which is especially good for business.

Anything that increases the distribution and comprehension of knowledge is a good thing, in my opinion, and when it concerns public relations it may also lead to increased economic benefits for the company, its employees and by extension society-at-large. Louhiala-Salminen and Kankaanranta (ibid., p. 267) stated in their study of internal communications that, 'it should not be concluded that only the use of English - or BELF - is the guarantee for successful international internal communication'. This is true also for external communication of all kinds, especially public relations and its modern-day transformative form. 
It seems relevant to consider how text may appear through the three-dimensional model for discourse analysis (Fairclough, 2001, pp. 20-30), even though it may be viewed as a challenging or novel interpretation, as various social conditions influence the construction of texts as well as their interpretation. Vuckovic (2008) also noted many factors co-exist that affect our ability to communicate competently, particularly over cultural grounds, and our collective perceptions act as an additional filter when viewed against communications styles, stated Singer (1987).

The challenge is to prove or support the hypothesis, even though it can never be wholly proven since 'we' are a combination of different cultures, values, beliefs and opinions. It may be a case of finding the so-called happy-medium, achieved through a careful consideration of language, audience goal and style - perceptual elements of basic journalism - but something that can be lacking in PR-led communications. I argue, in any case, the hypothesis is far from being disproved.

I believe this area requires more broader research. I hope to be able to consider this further and contribute to the body of knowledge and create a fieldimplementable framework for use. It could be instrumental to analyse possible differences between text produced within specific sectors and different countries to assess whether any relationships or commonalities exist. Deeper analysis into the relationship between the presence or not of specific factors may be illuminative, if it could be undertaken, such as if companies tend to do $X$ (that may affect their messaging) do they also do $\mathrm{Y}$ and $\mathrm{Z}$ at the same time, and more importantly why.

So what? At the risk of repeating myself ad nauseam, this is a piece of exploratory research to support or demolish a supposition. It has many restrictions and limitations. It cannot claim to be authoritative and greater research is necessary, both on a country-level and sector-level. The reality is that the 'answer' may never be answered, since there is no real way of stating with confidence how every recipient of messaging can react. The best is to hopefully find a way, if necessary, of improving how messaging is formulated with the objective of improving postreception activities. There is no explicit literature to support these limitations, since the limitations could effectively be limitless, due to the multitude of factors extant. 
I believe that this research has helped me validate my hypothesis and direct certain directions of thought, being supported by highlighted theories, practices and statements in the literature. It has convinced me that there be a wider problem and work is needed to back up this supposition.

Language matters, in other words, but less can be the equivalent of more. A little more thought may be beneficial. It may not require expertise, but a little finesse and polishing may potentially make a modicum of difference. This can lead to greater things being achieved. The solution I have in mind may not require radical change and it should be field-implementable: some may eventually seize upon it and prosper, as for the rest...

An exploratory thesis will, by its nature, invariably have errors, false leads and a degree of uncertainty. Some of the greatest criticism may fall on this author, whether now or in the future as additional research is conducted and findings refined. The second part of this quotation from Dale Carnegie seems apt to close this thesis, even though I hope that the future is not necessarily so dire - 'Develop success from failures. Discouragement and failure are two of the surest stepping stones to success'. 


\section{REFERENCES}

Adler, R.B. \& Proctor II, R.F. (2015) Looking out, looking in. 14th edn. Wadsworth/Cengage Learning: Boston, MA.

Akaka, M.A., Vargo, S.L. \& Lusch, R.F. (2013) 'The complexity of context: a service ecosystems approach for international marketing', Journal of Marketing Research, 21(4), 1-20. doi: 10.1509/jim.13.0032

Albaum, G.S. \& Duerr, E.E. (2011) International marketing and export management. 7th edn. Financial Times Prentice Hall: Harlow.

Aloni, M. \& Dekker, P. (2016) The Cambridge handbook of formal semantics. Cambridge University Press: Cambridge.

Apeltauer, E. \& Shaw, G. (1993) 'Multilingualism in a society of the future', European Journal of Education, 28(3), 273-294. doi: 10.2307/1503759

Baird, R., Baker, W. \& Kitazawa, M. (2014) 'The complexity of ELF', Journal of English as a Lingua Franca, 3(1), 171-196.

Balmer, J.M.T. \& Wilson, A.M. (2001) 'Understanding organisational culture and the implications for corporate marketing', European Journal of Marketing, 35(3/4), 353-367. doi: 10.1108/03090560110382066

Bartlett, C. \& Johnson, C. (1998) 'Is Business English a pidgin?', Language and intercultural Training, 16(1), 4-6.

Beat, J. et al. (2002) Scientific Writing: Easy when you know how. BMJ Books: London.

Bensing, J., Van Dulmen, S. \& Tates, K. (2003) 'Communication in context: new directions in communication research', Patient Education and Counseling, 50(1), 27-32. doi: 10.1016/S0738-3991(03)00076-4

Bentele, G. (1997) Public relations and reality: A contribution to a theory of public relation. In Public relations research: An international perspective, (Eds, Moss, D., MacManus, T. \& Verčič, D.) International Thomson Business Press, London, pp. 89-109.

Berry, R. (2012) English grammar: a resource book for students. Routledge: London.

Blythe, J. (2006) Essentials of marketing communications. 3rd edn. Pearson Education: London. 
Brannen, M.Y., Piekkari, R. \& Tietze, S. (2016) The multifaceted role of language in international business: Unpacking the forms, functions and features of a critical challenge to MNC theory and practice. In Language in International Business: Developing a field, (Eds, Brannen, M.Y. \& Mughan, T.) Springer, London.

Braun, S. (2004) The interplay between media, public relations, and society in Bulgaria. In Globalization: Chellenges \& opportunities for public relations (from the 7th International Public Relations Research conference, South Miami, FL, USA), Institute for Public Relations, Gainesville, FL, pp. 6-14.

Brechman, J., Lee, C.-j. \& Cappella, J.N. (2009) 'Lost in translation? A comparison of cancer-genetics reporting in the press release and its subsequent coverage in the press', Science Communication, 30(4), 453-474. doi:

$10.1177 / 1075547009332649$

Brookshire, R.H. \& Brundage, S.B. (2016) Writing scientific research in communication sciences and disorders. Plural Publishing: San Diego, CA.

Bruce, S. (2015) 'What is PR?: A definition for 2015', Influence. Available at: http://influence.cipr.co.uk/2015/01/05/pr-definition-2015/ (Accessed April 8, 2017).

Bryman, A. \& Bell, E. (2011) Business research methods. 3rd edn. Oxford University Press: Oxford.

Burnett, J. \& Moriarty, S. (1998) Introduction to marketing communication: an integrated approach. Prentice-Hall: Upper Saddle River, NJ.

Canagarajah, A.S. (2006) 'Negotiating the local in English as a lingua franca', Annual Review of Applied Linguistics, 26, 197-218. doi:

$10.1017 /$ S0267190506000109

Catenaccio, P. (2008) 'Press releases as a hybrid genre: Addressing the informative/promotional conundrum', Pragmatics, 18(1), 9. doi:

10.1075/prag.18.1.02cat

Chaffey, D. et al. (2006) Internet marketing: Strategy, implementation and practice. 3rd edn. Prentice Hall: Harlow.

Chall, J.S. (1947) 'This business of readability', Educational Research Bulletin, 26(1), 1-13.

Charles, M. (2007) 'Language matters in global communication: Article based on ORA lecture, October 2006', Journal of Business communication, 44(3), 260-282. doi: $10.1177 / 0021943607302477$

Chartered Institute of Public Relations (Undated) 'Our organisation', Available at: https://www.cipr.co.uk/content/our-organisation/ (Accessed April 17, 2017).

Chartered Institute of Public Relations (Undated) 'What is PR?', Available at: https://www.cipr.co.uk/content/careers-advice/what-pr/ (Accessed April 8, 2017). 
Christiansen, T.W. (2016) 'The Internet as a global speech community. Towards plurilingualisms and English Lingua Franca', Lingue e Linguaggi, 19, 77-96. doi: 10.1285/i22390359v19p77

Collins, H. (2010) Creative research: the theory and practice of research for the creative industries. AVA Publishing: London.

Cornelissen, J. (2004) Corporate communications: theory and practice. SAGE: London.

Crotty, M. (1998) The foundations of social research: Meaning and perspective in the research process. Sage Publications: London.

Cuypers, I., Ertug, G. \& Hennart, J.-F. (2015) 'The effects of linguistic distance and lingua franca proficiency on the stake taken by acquirers in cross-border acquisitions', Journal of International Business Studies, 46(4), 429-442. doi: $10.1057 /$ jibs.2014.71

Davies, M. (2015) Corpora: an introduction. In The Cambridge handbook of English corpus linguistics, (Eds, Biber, D. \& Reppen, R.) Cambridge University Press, Cambridge.

Davis, A.K., Piger, J.M. \& Sedor, L.M. (2012) 'Beyond the numbers: Measuring the information content of earnings press release language', Contemporary Accounting Research, 29(3), 845-868. doi: 10.1111/j.1911-3846.2011.01130.x

Davis, A.K., Piger, J.M. \& Sedor, L.M. (2006) 'Beyond the numbers: An analysis of optimistic and pessimistic language in earnings press releases', Available at: https://m.research.stlouisfed.org/wp/2006/2006-005.pdf/ (Accessed March 15, 2017).

Decke-Cornill, H. (2002) 'We would have to invent the language we are supposed to teach': The Issue of English as lingua franca in language education in Germany', Language Culture and Curriculum, 15(3), 251-263. doi: $10.1080 / 07908310208666649$

Dewey, M. (2007) 'English as a lingua franca and globalization: an interconnected perspective', International Journal of Applied Linguistics, 17(3), 332-354. doi: 10.1111/j.1473-4192.2007.00177.x

Dillard, J.P. \& Pfau, M. (2002) The persuasion handbook: Developments in theory and practice. Sage Publications: London.

Dixon, R.M.W. (1997) The rise and fall of languages. Cambridge University Press: Cambridge.

EF Education First (2016a) 'EF English Proficency Index', Available at: http://mediakey1.ef.com/_/ /media/centralefcom/epi/downloads/full-reports/v6/efepi-2016-english.pdf/ (Accessed May 15, 2017). 
EF Education First (2016b) 'EF EPI Country fact sheet: Suomi (Finland)', Available at: http://www.ef.fi/sitecore/_ /media/centralefcom/epi/downloads/factsheets/v6/ef-epi-country-fact-sheet-v6-fi-en.pdf/ (Accessed May 15, 2017).

Fairclough, N. (2001) Language and power. 2nd edn. Routledge: Abingdon.

Fairclough, N. (2010) Critical Discourse Analysis: The Critical Study of Language. 2nd edn. Routledge: New York.

Firth, A. (1996) 'The discursive accomplishment of normality: On 'lingua franca'English and conversation analysis', Journal of pragmatics, 26(2), 237-259. doi: 10.1016/0378-2166(96)00014-8

Flores, N. (2013) 'The unexamined relationship between neoliberalism and plurilingualism: A cautionary tale', TESOL Quarterly, 47(3), 500-520. doi: 10.1002/tesq.114

Ford, P.L. (1889) The prefaces, proverbs, and poems of Benjamin Franklin: Originally published in Poor Richard's Almanacs for 1733-1758. The Knickerbocker Press: New York, NY.

Franceschi, V. (2017) 'Plurilingual resources as an asset in ELF business interactions', Journal of English as a Lingua Franca, 6(1), 57-81.

Fry, E. (1968) 'A readability formula that saves time', Journal of Reading, 11(7), 513-578.

Fry, E. (1990) 'A readability formula for short passages', Journal of Reading, 33(8), 594-597.

Fry, E. (2002) 'Readability versus leveling', The Reading Teacher, 56(3), 286-291.

Gibson, D., Gonzales, J.L. \& Castanon, J. (2006) 'The importance of reputation and the role of public relations', Public Relations Quarterly, 51(3), 15.

Giles, H. (2016) Communication accommodation theory: negotiating personal relationships and social identities across contexts. Cambridge University Press: Cambridge.

Goldbort, R. (2006) Writing for science. Yale University Press: New Haven, CT.

Google (2016) 'Celebrating languages in the European Parliament', Available at: https://blog.google/topics/google-europe/celebrating-languages-in-european/ (Accessed May 2, 2017). 
Grunig, J.E. \& Hung-Baesecke, C.-J.F. (2015) Examining the influence of organization: Public relationships and organizational identification with a psychological group on institutional affiliation, behavioural intent, and evaluations of satisfaction. In Public relations as relationship management: A relational approach to the study and practice of public relations, (Eds, Ki, E.-J., Kim, J.-N. \& Ledingham, J.A.) Routledge, New York, NY.

Gubrium, J.F. \& Holstein, J.A. (1997) The new language of qualitative method. Oxford University Press: Oxford.

Guido, M.G. \& Seidlhofer, B. (2014) 'Introduction. English as a Lingua Franca: Theory and Practice', Textus, 27(1), 7-16. doi: 10.7370/77482

Hallahan, K. (1999) 'Seven models of framing: Implications for public relations', Journal of Public Relations Research, 11(3), 205-242. doi: 10.1207/s1532754xjprr1103_02

Hart, J. (2007) A writer's coach: The complete guide to writing strategies that work. Anchor: New York, NY.

Holtgraves, T.M. (2013) Language as social action: Social psychology and language use. Lawrence Erlbaum Associates: Mahwah, NJ.

House, J. (2002) Developing pragmatic competence in English as a lingua franca. In Lingua franca communication, (Eds, Knapp, K. \& Meierkord, C.) Peter Lang, London, pp. 245-267.

House, J. (2003a) 'English as a lingua franca: A threat to multilingualism', Journal of sociolinguistics, 7(4), 556-578. doi: 10.1111/j.1467-9841.2003.00242.x

House, J. (2003b) English as lingua franca and its influence on discourse norms in other languages. In Translation today: Trends and perspectives, (Eds, Anderman, G.M. \& Rogers, M.) Multilingual Matters, Tonawanda, NY.

Hsueh-Chao, M.H. \& Nation, P. (2000) 'Unknown vocabulary density and reading comprehension.', Reading in a foreign language, 13(1), 403-430.

Ihlen, Ø., Van Ruler, B. \& Fredriksson, M. (Eds.) (2009) Public relations and social theory: Key figures and concepts. Routledge, New York, NY.

Ingram, D.P. (2016) Public relations and external communications as an aid to export: Examination of leading companies in Western Finland. Bachelor's thesis, Centria University of Applied Sciences.

Järvinen, K. (2017) Voice characteristics in speaking a foreign language: A study of voice in Finnish and Englis as L1 and L2. Doctoral thesis, University of Tampere.

Jenkins, J. (2009) 'English as a lingua franca: Interpretations and attitudes', World Englishes, 28(2), 200-207. doi: 10.1111/j.1467-971X.2009.01582.x 
Jenkins, J. (2015) 'Repositioning English and multilingualism in English as a Lingua Franca', Englishes in Practice, 2(3), 49-85. doi: 10.1515/eip-2015-0003

Jenkins, J., Cogo, A. \& Dewey, M. (2011) 'Review of developments in research into English as a lingua franca', Language teaching, 44(03), 281-315. doi: $10.1017 /$ S0261444811000115

Johanson, J. \& Vahlne, J.-E. (1977) 'The internationalization process of the firm- A model of knowledge development and increasing foreign market commitments', Journal of International Business Studies, 8(1), 23-32. doi: 10.1057/palgrave.jibs. 8490676

Johnson, P. (1981) 'Effects on reading comprehension of language complexity and cultural background of a text', TESOL quarterly, 15(2), 169-181. doi: $10.2307 / 3586408$

Kankaanranta, A. (2005) "Hej Seppo, could you pls comment on this!": Internal email communication in lingua franca English in a multinational company. University of Jyväskylä/Centre for Applied Language Studies: Jyväskylä.

Kato, Y., Scott, D.J. \& Kato, S. (2010) The influence of intimacy and gender on emotions in mobile phone email. In Affective computing and interaction: Psychological, cognitive and neuroscientific perspectives, (Eds, Gökçay, D. \& Yildirim, G.) Information Science Reference, Hershey, NY.

Knickerbocker, H., Johnson, R.L. \& Altarriba, J. (2015) 'Emotion effects during reading: Influence of an emotion target word on eye movements and processing', Cognition and Emotion, 29(5), 784-806.

Kotler, P. \& Armstrong, G. (2010) Principles of Marketing. 13th global edn. Pearson: Upper Saddle River, NJ.

Kotler, P. et al. (2009) Marketing Management. 1st European edn. Pearson Education: London.

LaRocque, P. (2003) The concise guide to copy editing: Preparing written work for readers. Marion Street Press: Portland, OR.

Learmonth, M. (2008) 'Speaking out: evidence-based management: a backlash against pluralism in organizational studies', Organization, 15(2), 283-291. doi: $10.1177 / 1350508407087763$

Lin, C.A. (1993) 'Cultural differences in message strategies: A comparison between American and Japanese TV commercials', Journal of Advertising Research, 33(4), 40-49.

Louhiala-Salminen, L. \& Charles, M. (2007) English as the lingua franca of international business communication: Whose English? What English? In Intercultural and international business communication, (Eds, Palmer-Silveira, J.C., Ruiz-Garrido, M.F. \& Fortanet-Gomez, I.) Peter Lang, Bern, pp. 27-54. 
Louhiala-Salminen, L. \& Kankaanranta, A. (2012) 'Language as an issue in international internal communication: English or local language? If English, what English', Public Relations Review, 38(2), 262-269.

Maat, H.P. (2007) 'How promotional language in press releases is dealt with by journalists: genre mixing or genre conflict', The Journal of Business Communication (1973), 44(1), 59-95. doi: 10.1177/0021943606295780

Maat, H.P. (2008) 'Editing and genre conflict: How newspaper journalists clarify and neutralize press release copy', Pragmatics, 18(1), 87-113. doi: 10.1075/prag.18.1.05pan

Maat, H.P. \& de Jong, C. (2013) 'How newspaper journalists reframe product press release information', Journalism, 14(3), 348-371. doi: $10.1177 / 1464884912448914$

Maclean, D. (2006) 'Beyond English: Transnational corporations and the strategic management of language in a complex multilingual business environment', Management Decision, 44(10), 1377-1390. doi: 10.1108/00251740610715704

Mauranen, A. (2006) 'Signaling and preventing misunderstanding in English as lingua franca communication', International Journal of the Sociology of Language, 177, doi: 10.1515/IJSL.2006.008

McCallum, D.R. \& Peterson, J.L. (1982) 'Computer-based readability indexes', Proceedings of the ACM'82 Conference, 44-48.

McKie, D. \& Heath, R.L. (2016) 'Public relations as a strategic intelligence for the 21st century: Contexts, controversies, and challenges', Public Relations Review, 42(2), 298-305. doi: 10.1016/j.pubrev.2015.04.003

McLaren, Y. \& Gurău, C. (2005) 'Characterising the Genre of the Corporate Press Release.', LSP and Professional Communication, 5(1).

McLaughlin, G.H. (1969) 'SMOG grading: A new readability formula', Journal of Reading, 12(8), 639-646.

McWhorter, J. (2007) Language interrupted: Signs of non-native acquisition in standard language grammars. Oxford University Press: Oxford.

Meade, C.D. \& Smith, C.F. (1991) 'Readability formulas: cautions and criteria', Patient Education and Counseling, 17(2), 153-158. doi: 10.1016/07383991(91)90017-Y

Millot, P. (2017) 'Inclusivity and exclusivity in English as a Business Lingua Franca: The expression of a professional voice in email communication', English for Specific Purposes, 46, 59-71. doi: 10.1016/j.esp.2016.12.001 
Moingeon, B. \& Soenen, G.B. (2002) Corporate and organizational identities: integrating strategy, marketing, communication and organizational perspectives. Psychology Press: London.

Mosenthal, P.B. \& Kirsch, I.S. (1998) 'A new measure for assessing document complexity: The PMOSE/IKIRSCH document readability formula', Journal of Adolescent \& Adult Literacy, 41(8), 638-657.

Mundt, K. \& Groves, M. (2016) 'A double-edged sword: the merits and the policy implications of Google Translate in higher education', European Journal of Higher Education, 6(4), 387-401. doi: 10.1080/21568235.2016.1172248

Nakata, C. \& Huang, Y. (2005) 'Progress and promise: The last decade of international marketing research', Journal of Business Research, 58(5), 611-618. doi: 10.1016/j.jbusres.2003.10.001

NASDAQ OMX Helsinki (2013) 'Rules of the exchange 2 October 2013: unofficial translation', Available at:

http://www.nasdaqomx.com/digitalAssets/87/87944_rules-of-theexchange_02102013.pdf/ (Accessed May 13, 2017).

Newsom, D. \& Haynes, J. (2008) Public relations writing: Form \& style. 8th edn. Cengage Learning: Belmont, CA.

Nikko, T. (2009) Dialogic construction of understanding in cross-border corporate meetings. PhD thesis, Helsinki School of Economics. Available at:

http://urn.fi/URN:ISBN:978-952-488-342-9/ (Accessed April 10, 2017).

O'Regan, J.P. (2014) 'English as a lingua franca: An immanent critique', Applied Linguistics, 35(5), 533-552. doi: 10.1093/applin/amt045

OED Online (2017) 'Communication, n.', Available at: (paywall) (Accessed May 2, 2017).

OED Online (2015) 'Public relations, n.', Available at: (paywall) (Accessed December 15, 2015).

Okazaki, S., Mueller, B. \& Taylor, C.R. (2010) 'Measuring soft-sell versus hard-sell advertising appeals', Journal of Advertising, 39(2), 5-20. doi: 10.2753/JOA00913367390201

Owen, J. (2017) 'Reports of 'death of the press release' greatly exaggerated, PRWeek analysis finds', PRWeek. Available at: http://www.prweek.com/article/1427345/reports-death-press-release-greatlyexaggerated-prweek-analysis-finds/ (Accessed May 14. 2017).

Palmer-Silveira, J.C., Ruiz-Garrido, M.F. \& Fortanet Gómez, I. (2007) Facing the future of intercultural and international business communications. In Intercultural and international business communication, (Eds, Palmer-Silveira, J.C., RuizGarrido, M.F. \& Fortanet-Gomez, I.) Peter Lang, Bern, pp. 9-24. 
Pennebaker, J.W., Mehl, M.R. \& Niederhoffer, K.G. (2003) 'Psychological aspects of natural language use: our words, our selves', Annual review of psychology, 54(1), 547-577.

Pennycook, A. (2008) 'English as a language always in translation', European Journal of English Studies, 12(1), 33-47.

Perfetti, C. (2007) 'Reading ability: Lexical quality to comprehension', Scientific studies of reading, 11(4), 357-383. doi: 10.1080/10888430701530730

Phillipson, R. (2001) 'English for globalisation or for the world's people', International Review of Education, 47(3), 185-200. doi: 10.1023/A:1017937322957

Pickering, L. (2006) 'Current research on intelligibility in English as a lingua franca', Annual Review of Applied Linguistics, 26, 219-233. doi: $10.1017 /$ S0267190506000110

Pickton, D. \& Broderick, A. (2005) Integrated Marketing Communications. Second edition. Prentice Hall: Harlow.

Piekkari, R., Welch, D. \& Welch, L.S. (2014) Language in international business: The multilingual reality of global business expansion. Edward Elgar Publishing: Cheltenham.

Planken, B. (2012) The changing landscape of business communication: Developments and directions in research. In The language factor in international business: New perspectives on research, teaching and practice, (Eds, Heynderickx, P. et al.) Peter Lang, Bern, pp. 17-40.

Prime Minister's Office Finland (2017) 'Government convenes for a historic public session as part of the Parliament of the Future event', Available at:

http://vnk.fi/en/article/-/asset_publisher/valtioneuvosto-kokoontuu-historiallisestiyleison-eteen-tulevaisuuden-valtiopaivilla/ (Accessed May 2. 2017).

Raeymaeckers, K., Paulussen, S. \& De Keyser, J. (2012) A Survey of Professional Journalists in Flanders (Belgium). In The global journalist in the 21st century, (Eds, Weaver, D.H. \& Willnat, L.) Routledge, New York, NY.

Ravazzani, S. (2016) 'Exploring internal crisis communication in multicultural environments: A study among Danish managers', Corporate Communications: An International Journal, 21(1), 73-88. doi: 10.1108/CCIJ-02-2015-0011

Reay, T., Berta, W. \& Kohn, M.K. (2009) 'What's the evidence on evidence-based management', The Academy of Management Perspectives, 23(4), 5-18.

Redish, J.C. \& Selzer, J. (1985) 'The place of readability formulas in technical communication', Technical Communication, 32(4), 46-52. 
Redmond, M.V. \& Bunyi, J.M. (1993) 'The relationship of intercultural communication competence with stress and the handling of stress as reported by international students', International Journal of Intercultural Relations, 17(2), 235254. doi: 10.1016/0147-1767(93)90027-6

Robbins, T. (2003) Unlimited power: The new science of personal achievement. Free Press: New York, NY.

Ross, K. \& Nightingale, V. (2003) Media and audiences: new perspectives. Open University Press: Maidenhead.

Ryans Jr, J.K., Griffith, D.A. \& Steven White, D. (2003) 'Standardization/adaptation of international marketing strategy: Necessary conditions for the advancement of knowledge', International Marketing Review, 20(6), 588-603. doi:

$10.1108 / 02651330310505204$

Samovar, L.A. et al. (2013) Communication between cultures. 8th edn. Wadsworth: Boston, MA.

Schiffman, B., Nenkova, A. \& McKeown, K. (2002) 'Experiments in multidocument summarization', Proceedings of the second international conference on Human Language Technology Research, 52-58.

Seidlhofer, B. (2005) 'English as a lingua franca', ELT journal, 59(4), 339. doi: 10.1093/elt/cci064

Seidlhofer, B. (2007) Common property: English as a lingua franca in Europe. In International handbook of English language teaching: Part 1, (Eds, Cummins, J. \& Davison, C.) Springer Science \& Business Media, New York, NY.

Selzer, J. (1981) 'Readability is a four-letter word', Journal of Business Communication, 18(4), 23-34. doi: 10.1177/002194368101800403

Sewell Jr, W.H. (1992) 'A theory of structure: Duality, agency, and transformation', American journal of sociology, 98(1), 1-29.

Shrum, L.J. (2009) Media consumption and perceptions of social reality: effects and underlying processes. In Media effects: advances in theory and research, (Eds, Bryant, J. \& Oliver, M.B.) Routledge, London.

Simmons, S. (1991) How To Be the Life of the Podium. Amacom: New York, NY.

Singer, M.R. (1987) Intercultural communication: A perceptual approach. Prentice Hall: Upper Saddle River, NJ.

Skorczynska, H. \& Carrió-Pastor, M.L. (2015) 'Variation in general meaning keywords in press teleases from British and Spanish vompanies: Gaining feeper insights into corporate discourse', Procedia-Social and Behavioral Sciences, 198, 451-458. doi: 10.1016/j.sbspro.2015.07.465 
Smith, E.A. \& Kincaid, J.P. (1970) 'Derivation and validation of the automated readability index for use with technical materials', Human Factors: The Journal of the Human Factors and Ergonomics Society, 12(5), 457-564.

Sriramesh, K. (2009) 'The mass media and public relations: A conceptual framework for effective media relations in Asia', Asian Journal of Communication, 13(2), 1-20. doi: 10.1080/01292980309364836

Stacks, D.W. (2011) Primer of public relations research. 2nd edn. Guilford Press: New York, NY.

Stanton, S.J., Sinnott-Armstrong, W. \& Huettel, S.A. (2016) 'Neuromarketing: Ethical implications of its use and potential misuse', Journal of Business Ethics, 113. doi: 10.1007/s10551-016-3059-0

Steffensen, M.S., Joag-Dev, C. \& Anderson, R.C. (1979) 'A cross-cultural perspective on reading comprehension', Reading Research Quarterly, 10-29.

Stevens, K.T., Stevens, K.C. \& Stevens, W.P. (1992) 'Measuring the readability of business writing: The Cloze procedure versus readability formulas', Journal of Business Communication, 29(4), 367-382. doi: 10.1177/002194369202900404

Stubbs, M. (1990) Language in education. In An encyclopaedia of language, (Ed, Collinge, N.E.) Routledge, London.

Sumner, P. et al. (2016) 'Exaggerations and caveats in press releases and healthrelated science news', PloS one, 11(12), e0168217. doi:

10.1371/journal.pone.0168217

Superceanu, R. (2011) 'Intertextuality and informativity of press releases: Factors determining the communication between PR practitioner and journalist', PCTS Proceedings (Professional Communication \& Translation Studies), 4(1/2), 21-30.

Tench, R. et al. (2017) Communication excellence: How to develop, manage and lead exceptional communications. Palgrave Macmillan: Cham.

Valentini, C., Kruckeberg, D. \& Starck, K. (2016) 'The global society and its impact on public relations theorizing: Reflections on major macro trends', Central European Journal of Communication, 9(17), 229-246. doi: 10.19195/18995101.9.2(17).6

Valtioneuvosto (2017) 'Valtioneuvosto kokoontuu historiallisesti yleisön eteen Tulevaisuuden Valtiopäivillä', Available at: http://valtioneuvosto.fi/artikkeli/lasset_publisher/10616/valtioneuvosto-kokoontuu-historiallisesti-yleison-eteentulevaisuuden-valtiopaivilla/ (Accessed May 2, 2017).

Verčič, D., Zerfass, A. \& Wiesenberg, M. (2015) 'Global public relations and communication management: A European perspective', Public Relations Review, 41(5), 785-793. doi: 10.1016/j.pubrev.2015.06.017 
Volistedt, M. (2002) English as a language for internal company communications. In Lingua franca communication, (Eds, Knapp, K. \& Meierkord, C.) Peter Lang, London, pp. 87-107.

Vuckovic, A. (2008) 'Inter-cultural communication: a foundation of communicative action', Multicultural Education \& Technology Journal, 2(1), 47-59. doi: $10.1108 / 17504970810867151$

Waisbord, S. (2016) 'Communication studies without frontiers? Translation and cosmopolitanism across academic cultures', International Journal of Communication, 10, 868-886.

Welch, D.E., Welch, L.S. \& Marschan-Piekkari, R. (2001) 'The persistent impact of language on global operations', Prometheus, 19(3), 193-209.

White, C., Vanc, A. \& Stafford, G. (2010) 'Internal communication, information satisfaction, and sense of community: The effect of personal influence', Journal of Public Relations Research, 22(1), 65-84. doi: 10.1080/10627260903170985

Williams, K. (2003) Understanding media theory. Arnold/Hodder Education: London. 
This is a consolidation of two detailed qualitative interviews, being an adjustment to original research plans, which seeks to provide selected real-world observations about corporate PR functions and to assess opinions about key activities and operational attributes.

Both informants are senior executives holding communications roles within publicly listed Finnish companies that have extensive international activities. Informant 1's company is more product/solution-focussed in nature whilst Informant 2's company is more service-related. Direct quotations may be slightly amended to tidy up grammatical issues.

\section{Operation}

Both informants' companies have an English-first communications policy. 'English is the company language so we do everything in English first. If you think about, for instance, earnings announcements, they will be written in English and then translated into other languages,' said Informant 1, noting 'English is always the main language with everything basically and even if I'm heading Finland's communications, I will have to translate everything into English because our review rounds are such that basically everything needs to be in English. If I have something that would have only Finland in focus, which happens sometimes but not too often, then I would probably write it in Finnish because Finnish is my first language, but I would still write an English version as well. It takes sometimes a lot of time!'

Informant 2's company is similar in many ways to Informant 1's, although it can see internal decisions discussed and actioned in Finnish rather than English, dependent on the case and communications role, whilst English remains the official group language. In Informant 2's business unit there is less of an active, corporate newsroom-type approach, as noted with Informant 1's company, with more activity delegated to subsidiary companies to handle their own PR affairs.

Informant 2's business unit will primarily focus on financial and regulatory relations as its 'PR role', rather than announcing new products or services from subsidiary companies, unless the announcement is 'significant' that requires financial 
disclosure via a prescribed manner. In such cases, Informant 2's business unit may merely redistribute the messaging rather than be involved in its origination from the beginning.

Both companies must consider disclosure requirements (NASDAQ OMX Helsinki, 2013) with their PR activities.

\section{Message origination}

How a piece of PR messaging is originated can vary. In informant 1's company, proactive communication is usually topic-based and a decision is taken whether the type of messaging will vary by channel and if so separate strategies and implementations may be made around the same theme. Primarily this messaging is constructed in-house, with external agencies playing a subsidiary role at times. Informant 2's company's approach is different because of its aforementioned structure, so most messaging is constructed in a reactive manner in response to regulatory requirements. Proactive communication would be left to the subsidiary companies of Informant 2's company to undertake.

Co-workers and supervisor/managerial levels assist and validate proposals and output, with English being the operative language. Everything is produced 'English first' for Informant 1's company with any translation made after messaging has been finalised. It is less formulaic in Informant 2's case, dependent on the situation and who is involved with the activity, but it is not uncommon to author in an 'English first' approach for various internal reasons.

\section{Message review}

When a piece of messaging is prepared for release, the review process can be quite defined and varied, dependent on the scope of the announcement. Both companies have recourse to a legal check of messaging, although this is usually concerned with the disclosure requirements.

Informant 1 said that there are 'many eyes' on a piece of messaging, at different stages, to hopefully catch any errors and make it more intelligible, and whilst changes may be less drastic as the process develops, there is the potential for a 
very senior executive to have a diverging opinion at a later stage that could require major revisions or more - such a situation appears to be infrequent to Informant 1's company. There is a smaller group involved for review in Informant 2's company, partially due to the construction of the company and its policy of allowing subsidiary companies to handle their own PR. In Informant 2's case, the CEO may be involved as an interested party from an early stage. This is less common in Informant 1's case.

Every piece of communication must be finally reviewed by at least one nativeEnglish speaker, said Informant 1, even if English speakers have been also involved in the drafting process. 'It just needs to be perfect in a company like (Informant 1's employer), which is global. So, I can write English releases as well but someone makes sure in the end it is perfect in English as well,' said Informant 1. Informant 2's company would operate in a similar manner although it has less of a need for nuanced language, with most of its messaging being financial in nature and thus quite formulaic with figures doing most of the talking. Subsidiary companies of Informant 2's company may send pre-launch information to group as a courtesy, however, as Informant 2 noted: 'it's not that they need our permission to do that (release something) but it is a nice gesture to let the owner know what they are planning to do to get more customers...'

\section{Style differences}

When asked whether Informant 1's company makes a difference in its communications (and style of communication) to English-language messaging when the recipient is likely to be using ELF, Informant 1 confirmed that they did not, using instead the same English press release (or piece of communication) worldwide. It was not an issue that Informant 1 had considered before, but acknowledged that there may be some merit in considering a 'simpler' linguistic version in some circumstances and indicated that this may be something for future consideration.

Informant 2 was of a similar opinion, noting that in their case it was possibly less relevant as their business activities are focussed to countries and sectors where a good understanding of English may be a given, with any consumer messaging 
being handled by subsidiary companies who may also translate and originate different messaging if necessary. Informant 1's company may also translate some PR messaging for local markets, although this may be handled on a case-by-case basis and arranged through a local office.

\section{Understanding}

To both informants' knowledge edge there had not been any notable issues with linguistic misunderstanding of its external communications.

Informant 1 said that their company had no specific 'demographic target' for its reader in mind, other than perhaps a job position, such as a journalist who may have some specialist knowledge in the subject matter. This can also be extended to customers who would be expected to have a similar technical knowledge or better. By inference, therefore, any other audience to the communications may be secondary in nature and yet a target.

This can pose some challenges, noted Informant 1 , as at times '...our clients understand the (company products that can be quite technical) a lot better than a normal journalist and I guess some of our press releases can be quite cryptic and we try to make them understandable and we many times fail, which we realized by having journalists call us and asking what is this, what are you trying to say?'

PR messaging tends to be finalised around a press release at Informant 1's company and approved before derivatives may be considered, such as social media messaging. Where possible elements of ongoing or previous discussions are reused, even post-publication, in the case of newsletters or case studies. Different teams and operating divisions may be involved, but recourse to Informant 1 's team is available as necessary.

\section{Evaluation}

There is no specific quantitative or technical evaluation of the English-language messaging deployed in either respondent company during a message's construction. Informant 1 said that the only feedback they may get about a message from external parties comes post-release, perhaps as an 'in passing' 
comment from a journalist who did not understand some point, but this type of feedback is highly subjective and nothing that could be used as objective evaluation, other than a mere guide. Informant 2 had no explicit opinion on this matter. Any evaluation that does occur is thus restricted to traditional PR norms based around 'coverage' following a message's release.

\section{Gatekeeper role}

Both informants see a valuable, symbiotic role and place for PR and journalism both now and going forward.

The role of gatekeepers such as journalists remains an important one, said both informants, despite challenges facing the media industry-at-large and the trend for organizations to also communicate direct with their 'reader' publics too. Informant 1 said '...they are really important. I think we (at the company's communications department) are (company's) voice and we journalists to continue from there and be our voice in a way as well. So I think we are important as gatekeepers and they are really important too.'

Informant 2 sees an equal value with gatekeepers. 'I think journalists or media are one of our key groups because there are lots of information that we want our owners (the public) to know. It is like free publicity to get some messages in the media and then we have lots of private owners who don't own that many shares, but they are also important to us.'

\section{Social media and the future}

Social media has left its mark on Finnish companies in situations, although there can be differences in execution. Informant 1's company was more active, perhaps understandably so, whilst Informant 2's company had a more traditional approach and perhaps has not yet fully utilized the benefits of social media communications (subsidiary companies of Informant 2's company have, however, as befits their nature of business). Other online media is important for both companies, forming a primary method of communications. 


\section{Other observations}

Informant 1 had some personal views and observations about how other larger Finnish companies communicate, noting at times that it appears they fail to use a native speaker to check their communications. Informant 1 stated that many companies could improve how they undertake their global communications too.

Elements of the Finnish stereotypes about being selective with communications and not wishing to 'brag' about things having some validity at times in some situations, although it was not an overriding, disabling factor, noted both informants. 
The elements analysed were:

a. Weak verbs: Common English verbs with a vague meaning that may not trigger 'vivid imagery' and can require additional words to deliver meaning (Brookshire and Brundage, 2016, p. 152). Examples include 'to be', 'to have' and 'to give'.

b. Filler words: Unnecessary words that may be common in spoken language but add no meaning or relevance in written texts (Ibid.). Examples include 'very', 'much', 'possibly' and 'really'.

c. Nominalizations: Complex nouns that are formed from shorter verbs, adjectives and nouns (Ibid., p. 154). These can slow down comprehension. Examples include 'practicality', 'indication' and 'analysis'.

d. Entity substitutions: These are pronouns and vague determiners that are predominant in spoken language but in text can confuse a reader to the subject of the text (Schiffman et al., 2002). Examples include 'there', 'stuff' and 'thing'.

e. Negations: Words and prefixes that reverse the meanings of connected words which, at times, may slow a reader down and obscure a sentence's meaning (Aloni and Dekker, 2016, p. 472). Examples include 'not', 'nothing' and 'to undo'.

f. Noun clusters: This is a situation where three or more nouns appear consecutively, that may be difficult to comprehend (Beat et al., 2002, pp. 191-192). Examples include 'automated motor starting circuit' and 'penicillin skin test result group'.

g. Passive voice: A sentence or clause where the subject is the receiver of the main action, which in turn may appear weak or confusing to the reader (Brookshire and Brundage, 2016, pp. 161-165). Examples include 'is made', 'is being carried' and 'will be started'.

h. Modals: Verb modifiers that signify ability or necessity, being capable of weakening statements by making them appear uncertain or too radical (Berry, 2012, pp. 105-108). Examples include 'must', 'would' and 'may'.

i. Rare words: These words are not part of the 5000 most-frequently and commonly used words within the Corpus of Contemporary American English, a major linguistic corpus (Davies, 2015, p. 11). 
j. Extra-long sentences: Sentences with 40 or more words, which may create an impediment to understanding.

k. Extra-short sentences: Sentences with six or fewer words, which may lead to boredom, no clarity or add nothing of value.

I. Word count: The number of words excluding headlines and standardized 'boiler-plate' text at the end of the text. This can give an indication as to how concise and focused the messaging may be.

m. Words per sentence: A self-explanatory metric that also has relevance to items $\mathrm{j}$ and $\mathrm{k}$.

n. Readability: A computation of a comprehension level broadly corresponding to a U.S. school grade, based on the Flesch-Kincaid Grade Level formula (Stacks, 2011, p. 135).

o. Readability Average: A computation of an average of several different readability indices - Flesch-Kincaid Grade Level formula (Ibid.), Gunning Fog Index (Goldbort, 2006, p. 10), Coleman-Liau Index (McCallum and Peterson, 1982), SMOG Index (McLaughlin, 1969) and Automated Readability Index (Smith and Kincaid, 1970) - to give a balanced opinion of many metrics.

p. New Dale-Chall Readability Formula: A specific comprehension test arranged around fourth grade readers (and upwards) to indicate a relative simplicity and light comprehension (Fry, 2002).

q. Sentiment: A machine-interpreted metric to determine its perceived sentiment. It could be viewed as an experimental or guiding value, being a construction from one service provider, to indicate whether the text uses mostly positive language, negative language, or neutral language, although it cannot read the text to know if such usage is warranted, e.g. to announce lay-offs you are unlikely to use positive language. 


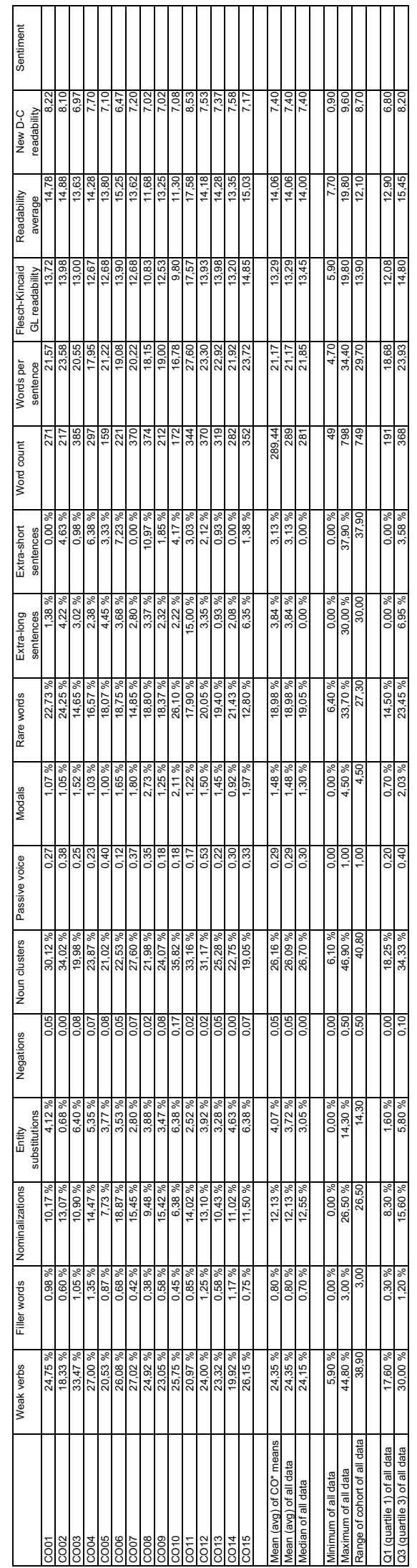

